\title{
EVALUATION OF PROLONGED ADMINISTRATION OF SULFA- QUINOXALINE ON THE HEPATORENAL PERFORMANCE OF BROILER CHICKENS WITH SPECIAL REFERENCE TO TISSUE RESIDUE LEVELS
}

\author{
D. A. Salem ${ }^{1}$, A. Z. Mahmoud ${ }^{2}$, Th. A. Ibrahim ${ }^{1}$, \\ M. Abd El-Naser ${ }^{1}$ and Eman, E. Gaber ${ }^{1}$ \\ 1-Dept. of Forensic Med. and Toxicology, Faculty of Vet. Med., Assiut University. \\ 2-Dept. of Pathology and Clinical Pathology, Faculty of Vet. Med., Assiut University.
}

\begin{abstract}
:
The effects of Sulfaquinoxaline (SQO) when used over a prolonged period for anticoccidial prophylaxis or as growth promotant on the hepatorenal performance were investigated. To determine the effect on the withdrawal period various tissue levels of SQO were also measured. A total of 180 (one day old) chicks were administered a prophylactic dose of SQO (1 g/L in drinking water) for 31 days. They were divided into three subgroups (60 chicks each). Group A1 given a prophylactic dose of SQO for two days followed by two days rest then given a prophylactic dose for another three days. Group A2 treated in the same way using therapeutic dose of SQO $(2 \mathrm{~g} / \mathrm{L})$ and group A3 treated also in the same way using a toxic dose of SQO $(8 \mathrm{~g} / \mathrm{L})$ of drinking water. Sixty one day old Ross chicks were used as control (group D).

Haematological studies revealed anemia. Impairment of hepatorenal performance was manifested by a significant decrease in the concentrations of total serum protein, as well as an increase in AST, ALT, creatinine, and uric acid. Chronic hepatopathy and nephropathy were observed in most chickens. Immunosuppression was indicated by hypoglobulinemia and depletion of lymphoid cell populations in the lymphoid organs. Higher concentrations of SQO residues were found in the kidney, followed by the liver and muscles. The SQO withdrawal period was extended from 15 to 18 days. It was concluded that the prolonged administration SQO has a deleterious effect on the hepatorenal functions, causes immunosuppression and lengthens the withdrawal period of the drug.
\end{abstract}




\section{INTRODUCTION :}

In Egypt, broiler chicken industry constitutes a very important source of animal protein. Infectious diseases remain a major problem facing poultry industry. Sulfonamides are consistently used for combating infectious diseases of poultry and as an antimicrobial growth-promoting factor. A number of sulfa have been implicated in poisoning of poultry, moreover the residues of sulfonamides on food of animal origin constitute hazards for public health. The experiment was conducted to evaluate the effect of sulfaquinoxaline (SQO) on the hepatorenal performance when used for a long period as a prophylaxis against coccidia or as a growth-promoting factor; SQO residue was also estimated to determine the withdrawal period.

\section{MATERIAL AND METHODS :}

A total of 180 one-day-old Ross chicks were used for this experiment. They were administered prophylactic dose of SQO (1 $\mathrm{g} / \mathrm{L}$ ) in drinking water for 31 days, then they were divided into three subgroups:

A1- (60 chicks) given prophylactic dose of SQO for two days, followed by two days rest then given prophylactic dose for another three days.

A2- (60 chicks) treated in the same way using therapeutic dose of SQO $(2 \mathrm{~g} / \mathrm{L})$ of drinking water.

A3- (60 chicks) treated in the same way using a toxic dose of SQO $(8 \mathrm{~g} / \mathrm{L})$ of drinking water.

D- 60 one day old Ross chicks were used as control which never administered SQO.
Slaughtering of chickens was begun at 31 days of age.

Blood, muscle, liver and kidney samples were collected at $12,24,48,96$ and $144 \mathrm{hrs}$ from the beginning of SQO administration (at 31 days of age). Similar types of samples were collected at $0,2,4,6,8,10,12,14,16$ and 18 days after stoppage of SQO administration.

Blood samples were used for erythrocytic count as followed by [1] and determination of $\mathrm{Hb}$ [2], PCV [3], MCV, MCH and MCHC [4]. Total protein was determined as [5], albumin [6], AST and ALT [7], gamaglutamate [8], uric acid [9] and creatinine in serum [10].

Liver, Kidney and muscle were used for determination of SQO residues by spectrophotometer as [11] and for histopathological studies.

\section{RESULTS :}

The results presented in tables $1 \& 2$ summaries the effects of SQO administration on the haematological picture of the exposed broiler chickens.

The effects of SQO on total protein, albumin, globulin and albumin/globulin ratio in the exposed birds were recorded in tables $3 \& 4$.

The results of enzymatic activities (AST, ALT, and $\gamma$-GT) and kidney function (uric acid and creatinine) were presented in tables 5\&6. The residual levels of SQO were recorded in tables $7 \& 8$.

The kidneys of group A1 and A2 chicken showed hypercellularity of some glomeruli (Fig. 1). The epithelium of the renal tubules especially those of the 
proximal convoluted tubules showed degenerative changes (Fig. 2). In addition, focal areas of fibroblastic reaction were seen in some cases of group $\mathrm{A2}$. Degenerative changes manifested by pyknosis, karryorhksis of the nuclei of the renal tubular epithelium were seen involving the whole nephron (Fig. 3).

The kidneys from group A3 showed prominent changes, but unlike the two previous groups, lesions of the renal tubular epithelium overcome those of the glomeruli and interstitial tissues. Lesions of the tubular epithelium include degeneration and necrosis (Fig. 4). The degenerative changes were manifested with severe vaculation (Fig. 5). However, the necrotic changes manifested by increase eosinophillia of the cytoplasm of these cells along with pyknosis and karryolsis of it's nucleus (Fig. 6) These changes were prominent the whole length of the nephron and especially it's lower part (collecting tubules).

Liver from chickens of group A1 showed histopathological changes of mild nature, a limited area of hepatic cell degeneration (Fig. 7). Liver from chickens of group A2 showed focal area of necrosis and degeneration were larger and extend to involve a relatively large area of hepatic tissues and were sometimes associated with focal area of lymphoid cell reaction (Fig. 8). Dilatation and congestion of hepatic sinusoids were seen, area of haemorrhage was sometimes observed (Fig. 9). The fibroblastic reaction was limited to portal area.

Liver from chickens of group A3 showed histopathological changes that involve the parenchymal cells, the hepatic vasculature and the bile duct system. In the parenchymal cells, a multiple focal areas of necrosis and degeneration were observed. The necrotic change of the hepatocytes was manifested by increase eosinophillia of the cytoplasm, a swelling of the cell and pyknosis of the nucleus (Fig. 10). However, the degenerative changes were manifested by vaculation and cytoplasmlysis with only a remnant of cytoplasm appear in the cell. Numerous numbers of hepatic cells showed evidence of mitosis. Multiple focal areas of haemorrhage were infrequently observed in between the parenchymal cell of the liver (Fig. 11). Blood vessels in the portal tract were congested or some time filled with a proteinous material and red blood cells (Fig. 12). Some bile duct in the area of portal tract show papillary hyperplasia of it's lining epithelium (Fig. 13). Such bile duct were prominently dilated and showed increased number of lining epithelium.

Cell proliferation was always observed in the area of portal tract especially around the hyperplastic bile duct (Fig. 14). A focal area of lymphoid and mononuclear cell reaction was observed in most cases (Fig. 15). Sometime these areas replace a massive number of hepatic cells (Fig. 16).

Micromorphological changes of the spleen from chickens of group A1 were limited to a very mild depletion of lymphoid cell population of the white pulp (Fig. 17). Histopathological examination of spleen of chickens from group A2 revealed moderate to sever exhaustion of lymphoid cell population of the splenic follicle (Fig. 18). The white pulp of the spleen from group A3 chickens showed prominent histopathological necrosis of hyaline type (Fig. 19). These necrotic changes involve the splenic 
follicle partially or completely. Histopathological examination of bursa fabricius from chickens of groups A1 and A2 revealed only decrease in the number of lymphoctyes observed mostly in the central part of the follicle. Fibrotic changes were not observed in the bursa in these groups.

Histopathological examination of the bursa of fabricius from chickens of group A3 revealed sever changes which involved the bursal follicle. In some cases the bursal follicle was partially or completely necrosed (Fig. 20\&21). The necrotic lymphoid cells were replaced with esinophillic cellular matrix. Necrosis and lysis of lymphoid cells were evident by the presence of empty spaces, which appeared mostly at the periphery of the bursal follicles (Fig. 22). In most of all cases of this group both size and numbers of bursal follicles were prominently decrease if compared to the control group.

The lymphoid tissues of the duodenum, the cecal tonsils from chickens of group A2 showed mild to moderate depletion in the lymphoid cells, in some cases. Vaculation and necrosis of epithelium of the duodenum were sometimes seen (Fig. 23). Haemorrhage in the tissues of the intestine was sometimes detected in this group. Histopathological examination of the lymphoid population of the payr's patches of the duodenum and ceacal tonsils from chicken of group $A 3$ revealed sever decrease in the number of the lymphoid cells of these two organs. The lymphoid tissues of these two organs in A3 group were replaced partially or completely by fibrous connective tissues (Fig. 24). 
Table (1): Effects of sulphaquinoxaline administration on haematological picture of exposed broiler chickens.

\begin{tabular}{|c|c|c|c|c|c|c|c|}
\hline $\begin{array}{l}\text { Time } \\
\text { (hour) }\end{array}$ & Group & $\begin{array}{c}\text { RBCs } \\
\left(10^{6} / \mathrm{mm}^{3}\right)\end{array}$ & $\begin{array}{c}\text { Hb } \\
(\mathrm{g} / \mathrm{L})\end{array}$ & $\begin{array}{l}\text { PCV } \\
(\%)\end{array}$ & $\begin{array}{c}\text { MCV } \\
(\mathrm{Pg})\end{array}$ & $\begin{array}{c}\text { МCH } \\
(\mathrm{Pg})\end{array}$ & $\begin{array}{c}\text { МСHC } \\
(\%)\end{array}$ \\
\hline \multirow{4}{*}{12} & A1 & $1.3 \pm 0.14 * *$ & $7.5 \pm 0.99 * *$ & $31.5 \pm 0.65 * b$ & $237 \pm 25 * * b$ & $55.7 \pm 5.0 * * b$ & $24.1 \pm 82 * * b$ \\
\hline & $\mathbf{A 2}$ & $1.2 \pm 0.18 * *$ & $7.1 \pm 0.12 * *$ & $31.0 \pm 00 * \mathrm{c}$ & $246.2 \pm 15^{* *} \mathrm{c}$ & $57.0 \pm 4.0 * * \mathrm{c}$ & $22.8 \pm 37 * * c$ \\
\hline & A3 & $1.5 \pm 0.10 * *$ & $6.9 \pm 0.32 * *$ & $0.29 \pm 47 * * b c$ & $191.8 \pm 15 * * b c$ & $45 \pm 1.6 * * \mathrm{bc}$ & $23.7 \pm 1.4 * * b c$ \\
\hline & D & $2.6 \pm 0.12$ & $10 \pm 0$ & $0.33 \pm 0$ & $123.6 \pm 5.6$ & $37.3 \pm 1.76$ & $30.0 \pm 0$ \\
\hline \multirow{4}{*}{24} & A1 & $1.4 \pm 0.09 * *$ & $7.4 \pm 0.7 * *$ & $31.0 \pm 0 *$ & $213.0 \pm 15.0 * *$ & $48.3 \pm 3.3 * *$ & $23.2 \pm 25 * *$ \\
\hline & $\mathbf{A 2}$ & $1.5 \pm 0.17 * *$ & $7.2 \pm 0.2 * *$ & $31.0 \pm 0 *$ & $217 \pm 75^{* *}$ & $51.3 \pm 9.0 * * \mathrm{c}$ & $23.9 \pm 66 * *$ \\
\hline & $\mathbf{A 3}$ & $1.4 \pm 0.17 * *$ & $7.0 \pm 0 * *$ & $30.0 \pm 0 * *$ & $211.3 \pm 7.3 * *$ & $49.5 \pm 1.6 * \mathrm{c}$ & $23 \pm 0 * *$ \\
\hline & D & $2.7 \pm 0.2$ & $10.0 \pm 0$ & $36.3 \pm 0.33$ & $122.3 \pm 7.8$ & $36.3 \pm 2.7$ & $28.5 \pm 0.76$ \\
\hline \multirow{4}{*}{48} & A1 & $1.3 \pm 0.21 * *$ & $7.0 \pm 0 * *$ & $30.2 \pm 25 * b$ & $243 \pm 38.6 * * a b$ & $55.5 \pm 8.3 * * a b$ & $22.7 \pm 0.14 * *$ \\
\hline & A2 & $1.3 \pm 0.49 * *$ & $7.0 \pm 0 * *$ & $31 \pm 40 * \mathrm{c}$ & $232.7 \pm 22.9 * * \mathrm{ac}$ & $49.0 \pm 6.1 * * a$ & $22.1 \pm 12 * *$ \\
\hline & A3 & $1.3 \pm 0.4$ & $6.5 \pm 0.2 * *$ & $27.5 \pm 28 * * b c$ & $208 \pm 26.4 * * b c$ & $49.5 \pm 7.4 * * b$ & $23.5 \pm 0.64 * *$ \\
\hline & D & $2.5 \pm 0.21$ & $10.3 \pm 0.16$ & $36.6 \pm 7.0$ & $145.5 \pm 2.7$ & $39.7 \pm 5.5$ & $27.13 \pm 0.53$ \\
\hline \multirow{4}{*}{96} & A1 & $1.3 \pm 0.15 * *$ & $7.2 \pm 0.14$ & $30.5 \pm 0.28 * b$ & $224.5 \pm 15 * * b$ & $53.5 \pm 3.7 * *$ & $23.6 \pm 0.55 * *$ \\
\hline & $\mathbf{A 2}$ & $1.45 \pm 0.18 * *$ & $7.17 \pm 0.11 \% *$ & $30.5 \pm 28 * \mathrm{c}$ & $222.2 \pm 32 * * \mathrm{c}$ & $52.5 \pm 8.1 * *$ & $23.3 \pm 43^{* *}$ \\
\hline & $\mathbf{A} 3$ & $1.3 \pm 0.1 * *$ & $6.5 \pm 0.28 * * b$ & $26.7 \pm 0.25 * * b c$ & $211 \pm 20.5 * * b c$ & $51 \pm 3.7$ & $24.3 \pm 1 * *$ \\
\hline & D & $2.5 \pm 0.1$ & $10 \pm 0$ & $38.0 \pm 0$ & $148.3 \pm 9.0$ & $39.7 \pm 1.6$ & $26.2 \pm 0.66$ \\
\hline \multirow{4}{*}{144} & A1 & $1.04 \pm 0.16 *$ & $7.1 \pm 0.12 * *$ & $30 \pm 0 * b$ & $199 \pm 20.4 * * b$ & $47.4 \pm 0.4 * * b$ & $23.4 \pm 40 * * b$ \\
\hline & $\mathbf{A 2}$ & $1.5 \pm 0.17 * *$ & $7.0 \pm 0 * *$ & $31 \pm 00 * \mathrm{c}$ & $203.5 \pm 22.19 * * c$ & $46 . \pm 4.9 * * \mathrm{c}$ & $22.5 \pm 0 * *$ \\
\hline & A3 & $1.2 \pm 0.16^{*}$ & $7.3 \pm 0.14 * *$ & $26.7 \pm 0.25 * * b c$ & $229 \pm 27 * *$ bc & $62.1 \pm 7.4 * * b c$ & $21.2 \pm 0.47 * * b$ \\
\hline & D & $2.6 \pm 0.12$ & $10.3 \pm 0.33$ & $38.3 \pm 0.33$ & $144.3 \pm 7.3$ & $39.2 \pm 0.73$ & $26.66 \pm 1.2$ \\
\hline
\end{tabular}

*Significant at $\mathbf{p} \leq 0.05$ in comparison with the control.

$* *$ Significant at $\mathbf{p} \leq \mathbf{0 . 0 1}$ in comparison with the control.

The same letter indicates the significant difference between the subgroup. 
Table (2): Effect of sulphaquinoxaline administration on haematological picture of exposed chickens after the stoppage of treatment.

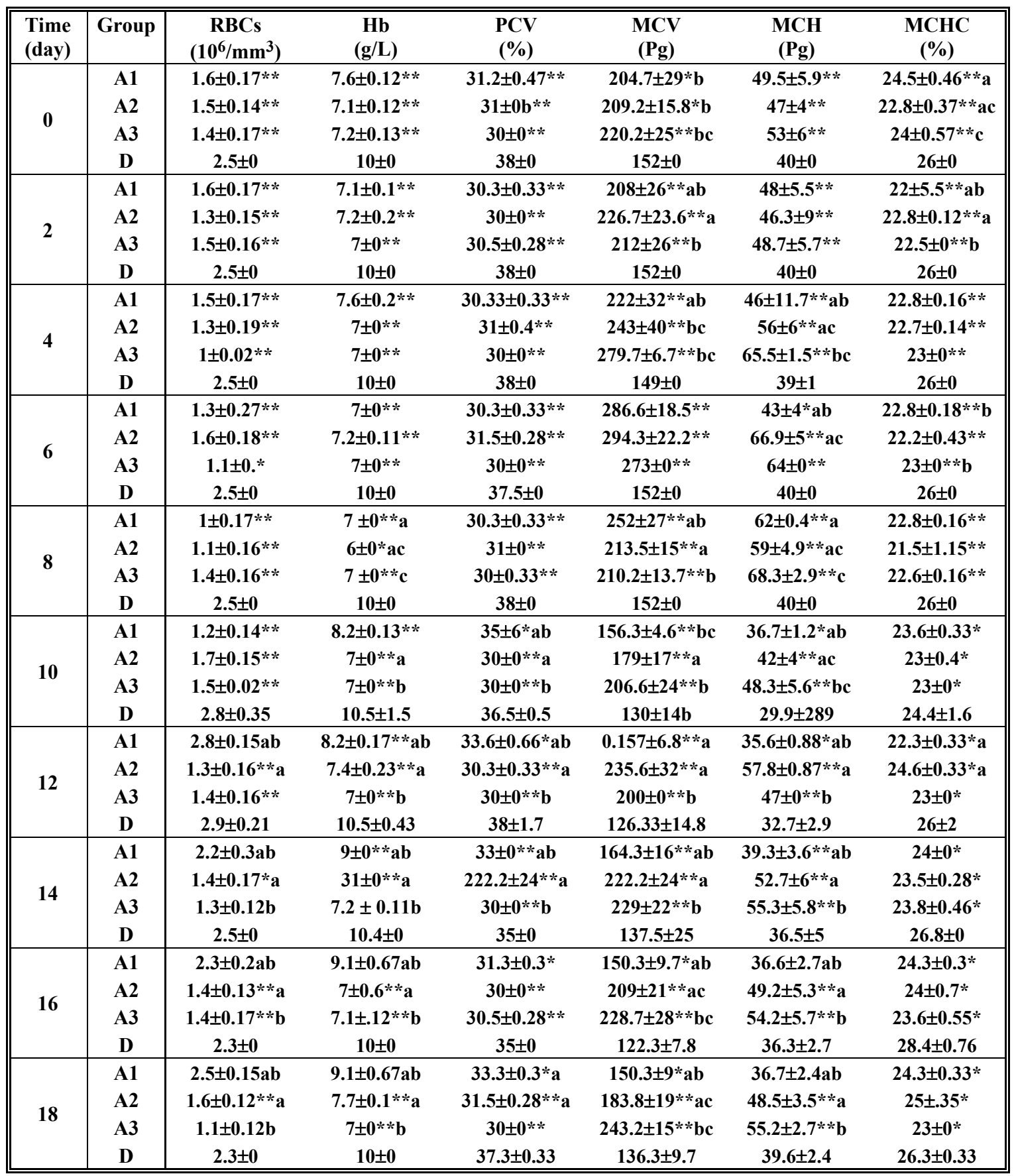

*Significant at $\mathbf{p} \leq \mathbf{0 . 0 5}$ in comparison with the control.

$* *$ Significant at $\mathbf{p} \leq \mathbf{0 . 0 1}$ in comparison with the control.

The same letter indicates the significant difference between the subgroup. 
Table (3): Effects of sulphaquinoxaline administration on total protein, Albumin Globulin, and A/G ratio of exposed birds during treatment.

\begin{tabular}{|c|c|c|c|c|c|}
\hline $\begin{array}{l}\text { Time } \\
\text { (hour) }\end{array}$ & Group & $\begin{array}{c}\text { Total protein } \\
(\mathrm{g} / \mathrm{L})\end{array}$ & $\begin{array}{c}\text { Albumin } \\
(\mathrm{g} / \mathrm{L})\end{array}$ & $\begin{array}{c}\text { Globulin } \\
(\mathrm{g} / \mathrm{L})\end{array}$ & $\mathrm{A} / \mathrm{G}$ ratio \\
\hline \multirow{4}{*}{12} & A1 & $40.6 \pm 1.9 * a b$ & $16.6 \pm 0.3 \mathrm{ab}$ & $23.8 \pm 0.7 b$ & $0.49 \pm 0.4 * a b$ \\
\hline & A2 & $45 \pm 3.2 \% \mathrm{ac}$ & $20 \pm 0.2 * * \mathrm{ac}$ & $25 \pm 0.7 * c$ & $0.8 \pm 0.05 * * a c$ \\
\hline & $\mathbf{A} 3$ & $46 \pm 1.2 * b c$ & $22 \pm 2.6 * * b c$ & $26.5 \pm 0.5 * * b c$ & $0.83 \pm 0.11 * * b c$ \\
\hline & D & $56.6 \pm 0.4$ & $16.36 \pm 0.4$ & $40 \pm 0.4$ & $0.4 \pm 0.11$ \\
\hline \multirow{4}{*}{24} & A1 & $44.5 \pm 1.2 * a b$ & $20.1 \pm 0.9$ & $22.3 \pm 1.7 * a b$ & $0.9 \pm 0.04 * * a b$ \\
\hline & A2 & $34.3 \pm 1.8 * * \mathrm{ac}$ & $14.4 \pm 1.7 * a$ & $20.9 \pm 1.4 * * \mathrm{ac}$ & $0.69 \pm 0.06 * * a c$ \\
\hline & A3 & $36.9 \pm 1.5^{* *} \mathrm{bc}$ & $20.1 \pm 2 *$ & $16.8 \pm 1.4 * * b c$ & $1.19 \pm 0.03 * * b c$ \\
\hline & D & $51.6 \pm 0.4$ & $17.3 \pm 0.4$ & $34.3 \pm 1.6$ & $0.50 \pm 0.05$ \\
\hline \multirow{4}{*}{48} & A1 & $46.0 \pm 0.5 * a b$ & $13.2 \pm 1.1 * a b$ & $32.7 \pm 0.6 * a b$ & $0.4 \pm 0 \mathrm{a}$ \\
\hline & A2 & $28.6 \pm 1.2 * * a c$ & $17.9 \pm 0.4 * \mathrm{ac}$ & $10.7 \pm 1 * * \mathrm{ac}$ & $16.7 \pm 0.07 * * a$ \\
\hline & A3 & $38.16 \pm 1.4^{* *}$ bc & $11 \pm 0.2 * b c$ & $27 \pm 2.2 * * \mathrm{bc}$ & $0.41 \pm 0.02^{* *}$ \\
\hline & D & $51.5 \pm 0.3$ & $18.2 \pm 0.2$ & $32.9 \pm 3$ & $0.55 \pm 0.03$ \\
\hline \multirow{4}{*}{96} & A1 & $45.3 \pm 0.5 * a b$ & $16.3 \pm 1$ * & $37.5 \pm 0.2 * a b$ & $0.43 \pm 0.03 * a b$ \\
\hline & $\mathbf{A} 2$ & $41.2 \pm 0.5 * * a$ & $16.4 \pm 0.4 *$ & $27.7 \pm 1 * * b c$ & $00.59 \pm 0.03 * * a c$ \\
\hline & $\mathbf{A 3}$ & $41.3 \pm 0.6 * * b$ & $16.4 \pm 0.1^{*}$ & $24.9 \pm 1 * * b c$ & $0.65 \pm 0.02 * * b c$ \\
\hline & D & $52.5 \pm 0.3$ & $17.7 \pm 0.2$ & $38 \pm 2.9$ & $0.45 \pm 0.09$ \\
\hline \multirow{4}{*}{144} & A1 & $49.2 \pm 0.5 * a b$ & $16 \pm 0.4^{* *} \mathrm{ab}$ & $23 \pm 0.6 * * a b$ & $0.69 \pm 0.06 * * a b$ \\
\hline & A2 & $43 \pm 3.1 * * a c$ & $18.4 \pm 0.5^{* * a c}$ & $24.8 \pm 3 * * \mathrm{ac}$ & $0.74 \pm 0.06 * * a c$ \\
\hline & A3 & $42.9 \pm 2.7^{* * b c}$ & $15.6 \pm 0.3 * * \mathrm{bc}$ & $28 \pm 3 * * \mathrm{bc}$ & $0.55 \pm 0.08 b c$ \\
\hline & D & $57 \pm 1.5$ & $21 \pm 0.5$ & $36 \pm 2$ & $0.58 \pm 0.08$ \\
\hline
\end{tabular}

*Significant at $\mathbf{p} \leq \mathbf{0 . 0 5}$ in comparison with the control.

$* *$ Significant at $\mathbf{p} \leq \mathbf{0 . 0 1}$ in comparison with the control.

The same letter indicates the significant difference between the subgroup. 
Table (4): Effects of sulphaquinoxaline administration on total protein, Albumin Globulin, and A/G ratio of exposed birds after the stoppage of treatment.

\begin{tabular}{|c|c|c|c|c|c|}
\hline $\begin{array}{l}\text { Time } \\
\text { (day) }\end{array}$ & Group & $\begin{array}{c}\text { Total protein } \\
(\mathrm{g} / \mathrm{L})\end{array}$ & $\begin{array}{c}\text { Albumin } \\
(\mathrm{g} / \mathrm{L})\end{array}$ & $\begin{array}{c}\text { Globulin } \\
(\mathrm{g} / \mathrm{L})\end{array}$ & $\mathrm{A} / \mathrm{G}$ ratio \\
\hline \multirow{4}{*}{$\mathbf{0}$} & A1 & $29.17 \pm 4.4 * * a b$ & $9.4 \pm 2 * * b$ & $19.7 \pm 5.0 * * a b$ & $0.47 \pm 0.07 a b$ \\
\hline & A2 & $16.7 \pm 2 * * a$ & $8.4 \pm 0.6 * *$ & $8.5 \pm 3 * *$ ac & $0.98 \pm 0.08 * * a$ \\
\hline & $\mathbf{A 3}$ & $17.9 \pm 2 * * b$ & $7.6 \pm 0.3 * * b$ & $13 \pm 4 * * \mathrm{bc}$ & $0.95 \pm 0.05 * * b$ \\
\hline & D & $51 \pm 1$ & $19.3 \pm 0.6$ & $31.7 \pm 1.6$ & $0.6 \pm 0.03$ \\
\hline \multirow{4}{*}{2} & A1 & $41.3 \pm 0.9 * * b$ & $19.9 \pm 0.4 * * b$ & $21.47 \pm 1.9 * * a b$ & $92 \pm 0.02 * * a$ \\
\hline & A2 & $39.7 \pm 2.3 * * \mathrm{c}$ & $18.8 \pm 1.4 * * \mathrm{c}$ & $17.1 \pm 3.4 * * a$ & $1.44 \pm 0.14 * * a c$ \\
\hline & A3 & $27.1 \pm 0.1 * * b c$ & $12.3 \pm 2 * *$ bc & $14.8 \pm 1 * * a b$ & $0.83 \pm 0.03 * * c$ \\
\hline & D & $51.25 \pm 0.3$ & $25.5 \pm 3.5$ & $25.75 \pm 3.7$ & $0.43 \pm 0.02$ \\
\hline \multirow{4}{*}{4} & A1 & $24.5 \pm 3.1 * * a b$ & $13.8 \pm 1.7 * * a b$ & $10.7 \pm 1.4 * * a b$ & $1.28 \pm 0.12 * * a b$ \\
\hline & $\mathbf{A 2}$ & $13.5 \pm 3.5^{* *} \mathrm{a}$ & $4.5 \pm 3.2 * * a$ & $9 \pm 1.6 * * a$ & $0.5 \pm 0 * a$ \\
\hline & A3 & $12 \pm 3 * * b$ & $4 \pm 5 * * b$ & $9 \pm 1 * * b$ & $0.44 \pm 0.04 b$ \\
\hline & D & $54.5 \pm 3.5$ & $15.5 \pm 2.5$ & $39 \pm 1$ & $0.39 \pm 0.3$ \\
\hline \multirow{4}{*}{6} & A1 & $33.9 \pm 3.2 * \% a b$ & $23.2 \pm 4.7 * * b$ & $19.6 \pm 1.6 * * a b$ & $0.72 \pm 0.07 * a b$ \\
\hline & A2 & $58 \pm 2.7 * * a$ & $21.8 \pm 1.3 * * c$ & $15.15 \pm 0.4 * * a$ & $1.43 \pm 0.43 * * a$ \\
\hline & A3 & $39.6 \pm 4.2 * * b$ & $14 \pm 2 * * \mathrm{bc}$ & $15.5 \pm 0.5 * * b$ & $1.48 \pm 0.81 * * b$ \\
\hline & D & $52 \pm 2$ & $28.8 \pm 0.2$ & $23.2 \pm 0.2$ & $0.56 \pm 0.03$ \\
\hline \multirow{4}{*}{8} & A1 & $33.9 \pm 2 * * a b$ & $17.6 \pm 0.3 * a b$ & $16 \pm 5 * * a b$ & $1.1 \pm 0.01 * * a b$ \\
\hline & A2 & $21.8 \pm 0.8 * * a c$ & $8.3 \pm 2 * * a c$ & $12.4 \pm 0.6 * * \mathrm{ac}$ & $0.4 \pm 0.04 a c$ \\
\hline & A3 & $31.7 \pm 0.4 * * b c$ & $22.5 \pm 4 * * b c$ & $9.2 \pm 2 * * \mathrm{bc}$ & $2.44 \pm 0.44 * * b c$ \\
\hline & D & $50.6 \pm 2$ & $27.2 \pm 0.2$ & $23.7 \pm 2$ & $0.51 \pm 0.03$ \\
\hline \multirow{4}{*}{10} & A1 & $34.6 \pm 8.2 * * a b$ & $17.7 \pm 16 a b$ & $16.9 \pm 1.9 * *$ & $1.04 \pm 0.04 * * a b$ \\
\hline & A2 & $31.8 \pm 4.6 * * a c$ & $13.8 \pm 2 * * a$ & $17.4 \pm 1.4 * *$ & $0.67 \pm 0.06 * a$ \\
\hline & A3 & $29.8 \pm 1.8^{* *} \mathrm{bc}$ & $11.1 \pm 0.8 * * b$ & $18.7 \pm 2.4 * * b$ & $0.7 \pm 0.03 b$ \\
\hline & D & $52.5 \pm 0.4$ & $16.36 \pm 0.4$ & $36 \pm 0.4$ & $0.45 \pm 0.05$ \\
\hline \multirow{4}{*}{12} & A1 & $42.5 \pm 2.2 * a b$ & $20.3 \pm 1.5 \mathrm{ab}$ & $22 \pm 1.7 * * a b$ & $0.92 \pm 0.03 * a b$ \\
\hline & A2 & $22.6 \pm 4 * * a c$ & $14.8 \pm 0.9 * * \mathrm{ac}$ & $5.6 \pm 2 \mathrm{ac}$ & $2 \pm 0.01 * * a$ \\
\hline & $\mathbf{A 3}$ & $17.2 \pm 2 * * \mathrm{bc}$ & $11.3 \pm 0.7 * * b c$ & $8 \pm 2 * * b c$ & $1.74 \pm 0.07 * * b$ \\
\hline & D & $54.6 \pm 0.4$ & $17.3 \pm 0.4$ & $37 \pm 0.4$ & $0.46 \pm 0.02$ \\
\hline \multirow{4}{*}{14} & A1 & $50 \pm 3 a b$ & $22.2 \pm 2.4 \mathrm{ab}$ & $28.06 \pm 0.7$ & $0.79 \pm 0.07 * a b$ \\
\hline & $\mathbf{A 2}$ & $47.2 \pm 2 \mathrm{a}$ & $19.9 \pm 2.9 \mathrm{ac}$ & $28.1 \pm 2$ & $58 \pm 0.05 * a c$ \\
\hline & A3 & $44.4 \pm 2 b$ & $16.5 \pm 2.6^{* *} \mathrm{~b}$ & $26.3 \pm 2 * *$ & $0.64 \pm 0.04 * b c$ \\
\hline & D & $56.5 \pm 0.33$ & $18.2 \pm 0.3$ & $38.3 \pm 1.2$ & $0.47 \pm 0.04$ \\
\hline \multirow{4}{*}{16} & A1 & $49 \pm 2.1 \mathrm{ab}$ & $15.7 \pm 0.8 \mathrm{ab}$ & $33.9 \pm 1.7 * * b$ & $0.46 \pm 0.04 * a b$ \\
\hline & A2 & $34.8 \pm 2.5 * * a$ & $16.1 \pm 2.6 * * a c$ & $18.7 \pm 1 * * c$ & $0.88 \pm 0.02 * a c$ \\
\hline & A3 & $32.8 \pm 0.3 * * b$ & $12.9 \pm 2 * * b c$ & $18.7 \pm 1.6 * * b c$ & $0.63 \pm 0.04 * b c$ \\
\hline & D & $56.6 \pm 0.3$ & $17.7 \pm 0.3$ & $39.5 \pm 3$ & $0.44 \pm 0.02$ \\
\hline \multirow{4}{*}{18} & A1 & $49.6 \pm 0.3 \mathrm{ab}$ & $22 \pm 2 a b$ & $28.1 \pm 2 * * a$ & $0.8 \pm 0 *$ \\
\hline & A2 & $30 \pm 1.7 \mathrm{a}$ & $14 \pm 2 * * a$ & $15.5 \pm 2.4 * * \mathrm{ac}$ & $0.9 \pm 0.9 *$ \\
\hline & $\mathbf{A 3}$ & $34 \pm 1.5 * b$ & $16.8 \pm 0.9 * * b$ & $17.3 \pm 3 * * \mathrm{c}$ & $0.85 \pm 0.08$ \\
\hline & D & $55 \pm 0.3$ & $21 \pm 0.6$ & $34 \pm 2$ & $0.61 \pm 0.1$ \\
\hline
\end{tabular}

$*$ Significant at $\mathbf{p} \leq \mathbf{0 . 0 5}$ in comparison with the control.

$* *$ Significant at $\mathbf{p} \leq \mathbf{0 . 0 1}$ in comparison with the control.

Same letter indicates the significant difference between the subgroup. 
Ass. Univ. Bull. Environ. Res. Vol. 3 No. 1, March 2000

Table (5): Effects of sulphaquinoxaline administration on enzymatic activities, uric acid and creatinine of exposed birds during treatment.

\begin{tabular}{|c|c|c|c|c|c|c|}
\hline \begin{tabular}{|l} 
Time \\
(hour)
\end{tabular} & Group & $\begin{array}{c}\text { AST } \\
(\text { IU/L) }\end{array}$ & $\begin{array}{c}\text { ALT } \\
(\text { IU/L) }\end{array}$ & $\begin{array}{l}\gamma-\mathbf{G T} \\
(\mathbf{I U} / \mathbf{L})\end{array}$ & $\begin{array}{c}\text { Uric acid } \\
(\mathrm{mg} / \mathrm{L})\end{array}$ & $\begin{array}{c}\text { Creatinine } \\
(\mathrm{mg} / \mathrm{L})\end{array}$ \\
\hline \multirow{4}{*}{12} & A1 & $16.6 \pm 3 * a b$ & $2 \pm 0.4 * * b$ & $2.13 \pm 0.33 * *$ & $68.5 \pm 0.64 * a b$ & $20.3 \pm 0.18$ \\
\hline & $\mathbf{A} 2$ & $17.9 \pm 1.2 * * \mathrm{a}$ & $2 \pm 0.6 *$ & $2.1 \pm 0.23 * *$ & $151.7 \pm 10.2 * * \mathrm{ac}$ & $23.5 \pm 1.8$ \\
\hline & A3 & $18.8 \pm 2.2 * * b$ & $2.8 \pm 0.3 * * b$ & $2.86 \pm 0.32 * *$ & $167 \pm 18 * * b c$ & $27.5 \pm 2.1$ \\
\hline & D & $13.1 \pm 0.9$ & $1.3 \pm 0.2$ & $1.22 \pm 0.67$ & $57.6 \pm 4$ & $20.1 \pm 1$ \\
\hline \multirow{4}{*}{24} & A1 & $14.8 \pm 1.2 * a b$ & $2.3 \pm 0.9 * *$ & $2 \pm 0.7 * * a b$ & $65.2 \pm 4.8 * b$ & $20 \pm 1$ \\
\hline & A2 & $21.6 \pm 2.8 * * a$ & $2.9 \pm 0.4 * *$ & $3.9 \pm 2.6 * * a$ & $66.7 \pm 3 * * c$ & $21 \pm 0.88$ \\
\hline & A3 & $22 \pm 1.1 * * b$ & $3 \pm 0.2$ & $3.4 \pm 0.62 * * b$ & $79.7 \pm 2.3 * * \mathrm{bc}$ & $20 \pm 1.2$ \\
\hline & D & $10.2 \pm 0.3$ & $0.7 \pm 0.02$ & $1.68 \pm 0.16$ & $36.6 \pm 1.7$ & $22.4 \pm 0.26$ \\
\hline \multirow{4}{*}{48} & A1 & $1.6 \pm 1.2 * \mathrm{ab}$ & $2.3 \pm 0.2 * *$ & $2.27 \pm 0.23 * *$ & $61 \pm 1.3 * a b$ & $17.5 \pm 1.6$ \\
\hline & A2 & $18.6 \pm 1.2 * * \mathrm{ac}$ & $2.7 \pm 0.8 * *$ & $2.34 \pm 0.18 * *$ & $77 \pm 1.4 * *$ ac & $18.5 \pm 2.2$ \\
\hline & A3 & $19.5 \pm 1.4 * * \mathrm{bc}$ & $3 \pm 0.7 * *$ & $2.58 \pm 0.74 * *$ & $87.7 \pm 0.14^{* *}$ bc & $21.5 \pm 3$ \\
\hline & D & $8.6 \pm 0.6$ & $1.3 \pm 0.2$ & $1.7 \pm 0.91$ & $54.5 \pm 1.1$ & $18.5 \pm 0.52$ \\
\hline \multirow{4}{*}{96} & A1 & $17.38 \pm 0.5 * a b$ & $2.2 \pm 0.8 * *$ & $2.61 \pm 0.35 * *$ & $85.2 \pm 7.9 * a b$ & $15.5 \pm 0.65$ \\
\hline & A2 & $22.35 \pm 0.5 * * \mathrm{ac}$ & $2.3 \pm 0.8 * *$ & $2.61 \pm 0.61 * *$ & $114.5 \pm 24 * *$ ac & $18.5 \pm 0.65$ \\
\hline & A3 & $26.7 \pm 0.4 * * b c$ & $2.5 \pm 0.2$ & $2.74 \pm 0.13 * *$ & $144 \pm 12 * *$ bc & $19.2 \pm 1.1$ \\
\hline & D & $8.6 \pm 1.4$ & $1.1 \pm 0.1$ & $1.39 \pm 0.19$ & $41 \pm 1$ & $16.6 \pm 0.12$ \\
\hline \multirow{4}{*}{144} & A1 & $18 \pm 0.4 * a b$ & $2 \pm 0.4 * *$ & $2.78 \pm 0.37 * *$ & 88.61.5*ab & $16.5 \pm 0.32$ \\
\hline & A2 & $22.5 \pm 0.31 * * a c$ & $2.5 \pm 0.4 * *$ & $2.08 \pm 0.25 * *$ & $195 \pm 1.5 * *$ ac & $18.5 \pm 1$ \\
\hline & A3 & $24.7 \pm 2 * * b c$ & $2.7 \pm 0.2 * *$ & $2.32 \pm 0.14 * *$ & $199 \pm 18 * * b c$ & $19 \pm 0$ \\
\hline & D & $8.8 \pm 0.9$ & $\mathbf{1} \pm \mathbf{0 . 0 7}$ & $1.2 \pm 0.21$ & $60.15 \pm 1.5$ & $16.1 \pm 0.38$ \\
\hline
\end{tabular}

*Significant at $\mathbf{p} \leq \mathbf{0 . 0 5}$ in comparison with the control.

$* *$ Significant at $\mathbf{p} \leq \mathbf{0 . 0 1}$ in comparison with the control.

The same letter indicates the significant difference between the subgroup. 
Table (6): Effects of sulphaquinoxaline administration on enzymatic activities, uric acid and creatinine of exposed birds after the stoppage of drug.

\begin{tabular}{|c|c|c|c|c|c|c|}
\hline $\begin{array}{l}\text { Time } \\
\text { (day) }\end{array}$ & Group & $\begin{array}{c}\text { AST } \\
\text { (IU/L) }\end{array}$ & $\begin{array}{c}\text { ALT } \\
\text { (IU/L) }\end{array}$ & $\begin{array}{l}\gamma \text {-GT } \\
(\mathbf{I U} / \mathbf{L})\end{array}$ & $\begin{array}{c}\text { Uric acid } \\
\text { (mg/L) }\end{array}$ & $\begin{array}{c}\text { Creatinine } \\
(\mathrm{mg} / \mathrm{L})\end{array}$ \\
\hline \multirow{4}{*}{$\mathbf{0}$} & A1 & $16.5 \pm 1.3 * b$ & $1.92 \pm 0.23 * b$ & $2.07 \pm 0.36 * *$ & $61.5 \pm 11$ & $8.5 \pm 0.63$ \\
\hline & A2 & $16.75 \pm 0.64 * * \mathrm{c}$ & $2.17 \pm 0.18 * *$ & $2.27 \pm 0.67 * *$ & $87.75 \pm 4.4$ & $10.3 \pm 1.2$ \\
\hline & $\mathbf{A 3}$ & $18.6 \pm 2.5^{* *} b c$ & $2.6 \pm 0.18$ & $2.51 \pm 0.18 * *$ & $96.25 \pm 8.8$ & $15 \pm 0.38$ \\
\hline & D & $15 \pm 1$ & $1.08 \pm 0.01$ & $1.66 \pm 0.04$ & $56 \pm 1.5$ & $17.2 \pm 0.6$ \\
\hline \multirow{4}{*}{2} & A1 & $18.7 \pm 0.43 * a b$ & $1.9 \pm 0.23 * b$ & $2.27 \pm 0.24 * *$ & $141.6 \pm 5$ & $15.5 \pm 0.65$ \\
\hline & $\mathbf{A 2}$ & $19.5 \pm 1 * * a$ & $2.7 \pm 0.62 * *$ & $2.35 \pm 0.25 * *$ & $105 \pm 5$ & $16.5 \pm 0.55$ \\
\hline & A3 & $20 \pm 1.9 * * b$ & $2.9 \pm 0.19 * * b$ & $2.55 \pm 0.34 * *$ & $162 \pm 11.6$ & $17.9 \pm 0.35$ \\
\hline & D & $14.5 \pm 3.5$ & $0.94 \pm 0.18$ & $1.94 \pm 0.01$ & $57 \pm 4$ & $16.5 \pm 0.55$ \\
\hline \multirow{4}{*}{4} & A1 & $16.35 \pm 1.3 * a b$ & $2.4 \pm 0.87 * *$ & $2.22 \pm 0.11 * *$ & $73.85 \pm 1.3$ & $15.8 \pm 0.2$ \\
\hline & $\mathbf{A 2}$ & $21.5 \pm 0.72 * * a c$ & $2.77 \pm 0.02 * *$ & $2.22 \pm 0.14 * *$ & $100 \pm 1.4$ & $17.5 \pm 2.2$ \\
\hline & $\mathbf{A 3}$ & $25.5 \pm 1.4 * * b c$ & $2.96 \pm 0.26 * *$ & $2.39 \pm 0.27 * *$ & $177 \pm 15.5$ & $19.5 \pm 2$ \\
\hline & D & $12.5 \pm 2$ & $0.93 \pm 0.04$ & $1.84 \pm 0.05$ & $60.4 \pm 5$ & $18.5 \pm 0.3$ \\
\hline \multirow{4}{*}{6} & A1 & $16 \pm 0.57 * b$ & $2.1 \pm 0.12 * *$ & $2.46 \pm 0.26^{* * *}$ & $104 \pm 13.4$ & $15.7 \pm 0.58$ \\
\hline & A2 & $16 \pm 1.57 * * \mathrm{c}$ & $2.72 \pm 0.88 * *$ & $2.5 \pm 0.36 * *$ & $127 \pm 13$ & $16.7 \pm 0.32$ \\
\hline & A3 & $19 \pm 0.57 * * b c$ & $2.75 \pm 0.18 * *$ & $2.5 \pm 0.18 * *$ & $128 \pm 14$ & $16.5 \pm 0.32$ \\
\hline & D & $15.6 \pm 2.1$ & $0.85 \pm 0.09$ & $1.5 \pm 0.02$ & $57 \pm 3$ & $18.8 \pm 0.31$ \\
\hline \multirow{4}{*}{8} & A1 & $17 \pm 0.57 * a b$ & $1.5 \pm 0.71 * a b$ & $1.9 \pm 0.26 * *$ & $127.9 \pm 18$ & $15.8 \pm 0.31$ \\
\hline & $\mathbf{A 2}$ & $18 \pm 0.57 * * a$ & $2.5 \pm 0.71 * * a$ & $2.9 \pm 0.31 * *$ & $144 \pm 25$ & $15.5 \pm 0.57$ \\
\hline & $\mathbf{A 3}$ & $18 \pm 0.57 * b$ & $2.9 \pm 0.98 * * b$ & $2.9 \pm 0.31 * *$ & $156 \pm 19$ & $15.8 \pm 0.88$ \\
\hline & D & $15.6 \pm 2.1$ & $0.85 \pm 0.09$ & $1.5 \pm 0.02$ & $57 \pm 3$ & $18.8 \pm 0.31$ \\
\hline \multirow{4}{*}{10} & A1 & $16.9 \pm 0.57$ & $2.44 \pm 0.71$ & $1.52 \pm 0.26^{*}$ & $144.34 \pm 25$ & $15.3 \pm 0.12$ \\
\hline & $\mathbf{A 2}$ & $18 \pm 0.94$ & $2.57 \pm 0.72$ & $2.6 \pm 0.35^{* *}$ & $157 \pm 30$ & $16.5 \pm 0.13$ \\
\hline & $\mathbf{A 3}$ & $18 \pm 1.3$ & $2.6 \pm 0.34$ & $2.7 \pm 0.17 * *$ & $166 \pm 6.9$ & $16 \pm 0.12$ \\
\hline & D & $15.5 \pm 2.3$ & $1.08 \pm 0.01$ & $1.66 \pm 0.04$ & $56 \pm 1.5$ & $18.5 \pm 0.6$ \\
\hline \multirow{4}{*}{12} & A1 & $15.49 \pm 1.3$ & $2.57 \pm 0.71$ & $2.47 \pm 0.24$ & $193.33 \pm 4.6$ & $9.5 \pm 0.53$ \\
\hline & A2 & $17 \pm 0.057$ & $2.78 \pm 0.25$ & $2.63 \pm 0.1$ & $155.5 \pm 8.6$ & $10.5 \pm 3$ \\
\hline & A3 & $19 \pm 0.57$ & $2.88 \pm 0.42$ & $2.74 \pm 0.98$ & $163 \pm 4.7$ & $12 \pm 2$ \\
\hline & D & $14.5 \pm 3.5$ & $0.94 \pm 0.18$ & $1.94 \pm 0.01$ & $57 \pm 4$ & $18.5 \pm 0.55$ \\
\hline \multirow{4}{*}{14} & A1 & $14.03 \pm 0.88$ & $2.57 \pm 0.71$ & $2.18 \pm 0.31$ & $140.3 \pm 2.6$ & $12.5 \pm 0.6$ \\
\hline & A2 & $18.3 \pm 0.53$ & $2.87 \pm 0.22$ & $2.6 \pm 1$ & $164 \pm 25$ & $12.4 \pm 0.63$ \\
\hline & A3 & $18.5 \pm 0.33$ & $2.92 \pm 0.21$ & $2.7 \pm 1$ & $165 \pm 15$ & $12.5 \pm 0.33$ \\
\hline & D & $12.5 \pm 2$ & $0.93 \pm 0.04$ & $1.84 \pm 0.25$ & $60 \pm 5$ & $18.5 \pm 0.3$ \\
\hline \multirow{4}{*}{16} & A1 & $15.9 \pm 1.8$ & $1.9 \pm 0.92$ & $2.26 \pm 0.25$ & $46 \pm 2$ & $12.7 \pm 0.32$ \\
\hline & A2 & $10.4 \pm 0.7$ & $2.1 \pm 0.37$ & $2.6 \pm 1.1$ & $74.5 \pm 5.8$ & $12.88 \pm 0.13$ \\
\hline & A3 & $12 \pm 0.47$ & $2.3 \pm 0.17$ & $2.7 \pm 1$ & $80.5 \pm 4$ & $13.5 \pm 0.41$ \\
\hline & D & $15.6 \pm 2$ & $0.93 \pm 0.11$ & $1.84 \pm 0.05$ & $60.4 \pm 5$ & $18.5 \pm 0.3$ \\
\hline \multirow{4}{*}{18} & A1 & $13.1 \pm 1.3$ & $1.3 \pm 0.15$ & $1.67 \pm 0.35$ & $42.5 \pm 5$ & $9.2 \pm 0.3$ \\
\hline & A2 & $13.4 \pm 0.39$ & $2.4 \pm 0.13$ & $1.99 \pm 0.37$ & $82 \pm 0.24$ & $12.5 \pm 0.5$ \\
\hline & $\mathbf{A 3}$ & $13.5 \pm 0.33$ & $2.5 \pm 0.1$ & $2 \pm 0.1$ & $100 \pm 0.33$ & $12.7 \pm 0.3$ \\
\hline & D & $1.56 \pm 2.1$ & $0.85 \pm 0.09$ & $1.5 \pm 0.2$ & $57 \pm 3$ & $18.8 \pm 0.31$ \\
\hline
\end{tabular}

*Significant at $\mathrm{p} \leq \mathbf{0 . 0 5}$ in comparison with the control.

$* *$ Significant at $\mathbf{p} \leq \mathbf{0 . 0 1}$ in comparison with the control.

Same letter indicates the significant difference between the subgroup. 
Table (7): Residual content of sulphaquinoxaline in tissues of birds during the drug administration.

\begin{tabular}{|c|c|c|c|c|}
\hline $\begin{array}{l}\text { Time } \\
\text { (hour) }\end{array}$ & Group & $\begin{array}{l}\text { Muscles } \\
\text { (mg/kg) }\end{array}$ & $\begin{array}{c}\text { Liver } \\
\text { (mg/kg) }\end{array}$ & $\begin{array}{l}\text { Kidney } \\
\text { (mg/kg) }\end{array}$ \\
\hline \multirow{4}{*}{12} & A1 & $3.7 \pm 0.28 \mathrm{ab}$ & $6.3 \pm 0.32 b$ & $12.4 \pm 0.27 \mathrm{~b}$ \\
\hline & A2 & $4.6 \pm 0.17 a$ & $6.8 \pm 0.28 c$ & $12.9 \pm 0.01 \mathrm{c}$ \\
\hline & $\mathbf{A 3}$ & $4.8 \pm 0.19 b$ & $7.5 \pm 0.69 b c$ & $13.2 \pm 0.21 b c$ \\
\hline & D & N.D. & N.D. & N.D. \\
\hline \multirow{4}{*}{24} & A1 & $6.8 \pm 0.22 b$ & $9 \pm 0.20 .24 a b$ & $15.3 \pm 0.23 \mathrm{ab}$ \\
\hline & A2 & $7.2 \pm 1 \mathrm{c}$ & $10.5 \pm 0.27$ & $16.7 \pm 0.4 \mathrm{ac}$ \\
\hline & A3 & $9.4 \pm 0.12 b c$ & $10.9 \pm 0.8 b$ & $18.8 \pm 0.19 b c$ \\
\hline & D & N.D. & N.D. & N.D. \\
\hline \multirow{4}{*}{48} & A1 & $8.6 \pm 0.25 a b$ & $11.6 \pm 0.24 a b$ & $18 \pm 0.38 a b$ \\
\hline & A2 & $9.8 \pm 0.33 a$ & $12.6 \pm 0.19 \mathrm{ac}$ & $19.1 \pm 0.39 \mathrm{a}$ \\
\hline & A3 & $10.7 \pm 0.9 b$ & $13.4 \pm 0.11 b c$ & $19.3 \pm 0.3 \mathrm{ab}$ \\
\hline & D & N.D. & N.D. & N.D. \\
\hline \multirow{4}{*}{96} & A1 & $6.4 \pm 0.19 \mathrm{ab}$ & $8.9 \pm 0.33 b$ & $14.2 \pm 0.5 b$ \\
\hline & A2 & $7.5 \pm 0.17 \mathrm{ac}$ & $9.7 \pm 0.34 \mathrm{c}$ & $14.9 \pm 0.49 \mathrm{c}$ \\
\hline & $\mathbf{A 3}$ & $8.4 \pm 0.1 b c$ & $10.5 \pm 0.4 b c$ & $17.3 \pm 0.14 b c$ \\
\hline & D & N.D. & N.D. & N.D. \\
\hline \multirow{4}{*}{144} & A1 & $9.9 \pm 0.33 \mathrm{ab}$ & $13 \pm 0.31 \mathrm{ab}$ & $19.8 \pm 0.1 \mathrm{ab}$ \\
\hline & A2 & $11.3 \pm 0.34 \mathrm{ac}$ & $14.5 \pm 0.11 \mathrm{ac}$ & $21.2 \pm 0.39 \mathrm{ac}$ \\
\hline & $\mathbf{A 3}$ & $12.6 \pm 0.16 b c$ & $15.7 \pm 0.2 \mathrm{bc}$ & $24.4 \pm 0.13 b c$ \\
\hline & D & N.D. & N.D. & N.D. \\
\hline
\end{tabular}

N.D. : Not detectable. 
Table (8): Residual content of sulphaquinoxaline in tissues of birds after the stoppage of drug administration.

\begin{tabular}{|c|c|c|c|c|}
\hline $\begin{array}{l}\text { Time } \\
\text { (day) }\end{array}$ & Group & $\begin{array}{c}\text { Muscles } \\
\text { (mg/kg) }\end{array}$ & $\begin{array}{c}\text { Liver } \\
\text { (mg/kg) }\end{array}$ & $\begin{array}{c}\text { Kidney } \\
\text { (mg/kg) }\end{array}$ \\
\hline \multirow{4}{*}{ 0 } & $\mathbf{A 1}$ & $5.8 \pm 1.1 \mathrm{ab}$ & $6.3 \pm 0.14 \mathrm{ab}$ & $9 \pm 0.33 a b$ \\
\hline & $\mathbf{A 2}$ & $6.4 \pm 0.13 \mathrm{ab}$ & $8.1 \pm 0.2 \mathrm{a}$ & $10.7 \pm 0.2 \mathrm{ac}$ \\
\hline & $\mathbf{A 3}$ & $7.5 \pm 0.23 \mathrm{bc}$ & $8.16 \pm 0.12 b$ & $12.9 \pm 0.46 b c$ \\
\hline & D & N.D. & N.D. & N.D. \\
\hline \multirow{4}{*}{2} & A1 & $4.4 \pm 0.2 \mathrm{ab}$ & $5.3 \pm 0.21 \mathrm{ab}$ & $8.5 \pm 0.24 a b$ \\
\hline & A2 & $5.8 \pm 0.2 \mathrm{ac}$ & $7.4 \pm 0.19 \mathrm{a}$ & $10.2 \pm 0.29 \mathrm{a}$ \\
\hline & A3 & $6.8 \pm 0.24 b c$ & $7.5 \pm 0.1 b$ & $12.5 \pm 0.56 \mathrm{bc}$ \\
\hline & D & N.D. & N.D. & N.D. \\
\hline \multirow{4}{*}{4} & A1 & $41 \pm 0.44 b$ & $4.8 \pm 0.12 \mathrm{ab}$ & $8 \pm 0.19 a b$ \\
\hline & $\mathbf{A 2}$ & $5 \pm 0.23$ & $6.4 \pm 0.26 \mathrm{a}$ & $9.3 \pm 0.31 \mathrm{ac}$ \\
\hline & $\mathbf{A 3}$ & $5.8 \pm 0.22 b$ & $6.57 \pm 0.17 b$ & $11.4 \pm 0.32$ \\
\hline & D & N.D. & N.D. & N.D. \\
\hline \multirow{4}{*}{6} & A1 & $3.1 \pm 0.1 \mathrm{ab}$ & $4.6 \pm 0.24 b$ & $7.5 \pm 0.2 \mathrm{~b}$ \\
\hline & A2 & $4.3 \pm 0.18 \mathrm{a}$ & $4.9 \pm 0.2 \mathrm{c}$ & $7.7 \pm 0.7 \mathrm{c}$ \\
\hline & A3 & $5.1 \pm 0.12 b$ & $5.9 \pm 0.7 \mathrm{bc}$ & $10.4 \pm 0.25 b c$ \\
\hline & D & N.D. & N.D. & N.D. \\
\hline \multirow{4}{*}{8} & A1 & $2.9 \pm 0.1 \mathrm{ab}$ & $4.4 \pm 0.24 \mathrm{ab}$ & $7.1 \pm 0.2 \mathrm{ab}$ \\
\hline & $\mathbf{A 2}$ & $4.1 \pm 0.33 a$ & $5.6 \pm 0.18 a$ & $8 \pm 0.31 \mathrm{ac}$ \\
\hline & A3 & $4.6 \pm 0.2 b$ & $5.7 \pm 0.26 b$ & $9.9 \pm 0.85 b c$ \\
\hline & D & N.D. & N.D. & N.D. \\
\hline \multirow{4}{*}{10} & A1 & $2.75 \pm 0.25 b$ & $3.5 \pm 0.21 b$ & $7.7 \pm 0.67 \mathrm{ab}$ \\
\hline & A2 & $3.6 \pm 0.17$ & $3.8 \pm 0.28 c$ & $8.9 \pm 0.1 \mathrm{ac}$ \\
\hline & $\mathbf{A 3}$ & $3.45 \pm 0.33 b$ & $4.9 \pm 0.15 \mathrm{bc}$ & $9.6 \pm 0.5 b c$ \\
\hline & D & N.D. & N.D. & N.D. \\
\hline \multirow{4}{*}{12} & A1 & $2.3 \pm 0.16 b$ & $3.15 \pm 0.14 b$ & $5.2 \pm 0.12 \mathrm{ab}$ \\
\hline & A2 & $2.5 \pm 1$ & $3.5 \pm 0.27 \mathrm{c}$ & $6.7 \pm 0.4 \mathrm{ac}$ \\
\hline & $\mathbf{A} 3$ & $2.88 \pm 0.16 b$ & $4.8 \pm 0.15 b c$ & $8.9 \pm 0.25 b c$ \\
\hline & D & N.D. & N.D. & N.D. \\
\hline \multirow{4}{*}{14} & A1 & $1.9 \pm 0.01 b$ & $2.7 \pm 0.12 b$ & $3.9 \pm 0.26 \mathrm{ab}$ \\
\hline & A2 & $1.9 \pm 0.5 \mathrm{c}$ & $3.2 \pm 0.15$ & $4.5 \pm 0.22 \mathrm{ac}$ \\
\hline & $\mathbf{A 3}$ & $2.45 \pm 0.6 b c$ & $3.77 \pm 0.14 b$ & $7.28 \pm 0.4 b c$ \\
\hline & D & N.D. & N.D. & N.D. \\
\hline \multirow{4}{*}{16} & A1 & $1 \pm 0.33$ & $1.22 \pm 0.29 \mathrm{~b}$ & $1.8 \pm 0 b$ \\
\hline & A2 & $1 \pm 0.12$ & $.155 \pm 0.12$ & $1.99 \pm 0 \mathrm{c}$ \\
\hline & $\mathbf{A 3}$ & $1 \pm 0.28$ & $2.3 \pm 0.3 b$ & $3.2 \pm 0.8 \mathrm{bc}$ \\
\hline & D & N.D. & N.D. & N.D. \\
\hline \multirow{4}{*}{18} & A1 & N.D. & N.D. & N.D. \\
\hline & $\mathbf{A 2}$ & N.D. & $1.4 \pm 0.19$ & $1.8 \pm 0$ \\
\hline & $\mathbf{A 3}$ & N.D. & $1.6 \pm 0.28$ & $1.9 \pm$ \\
\hline & D & N.D. & N.D. & N.D. \\
\hline
\end{tabular}

N.D. : Not detectable. 


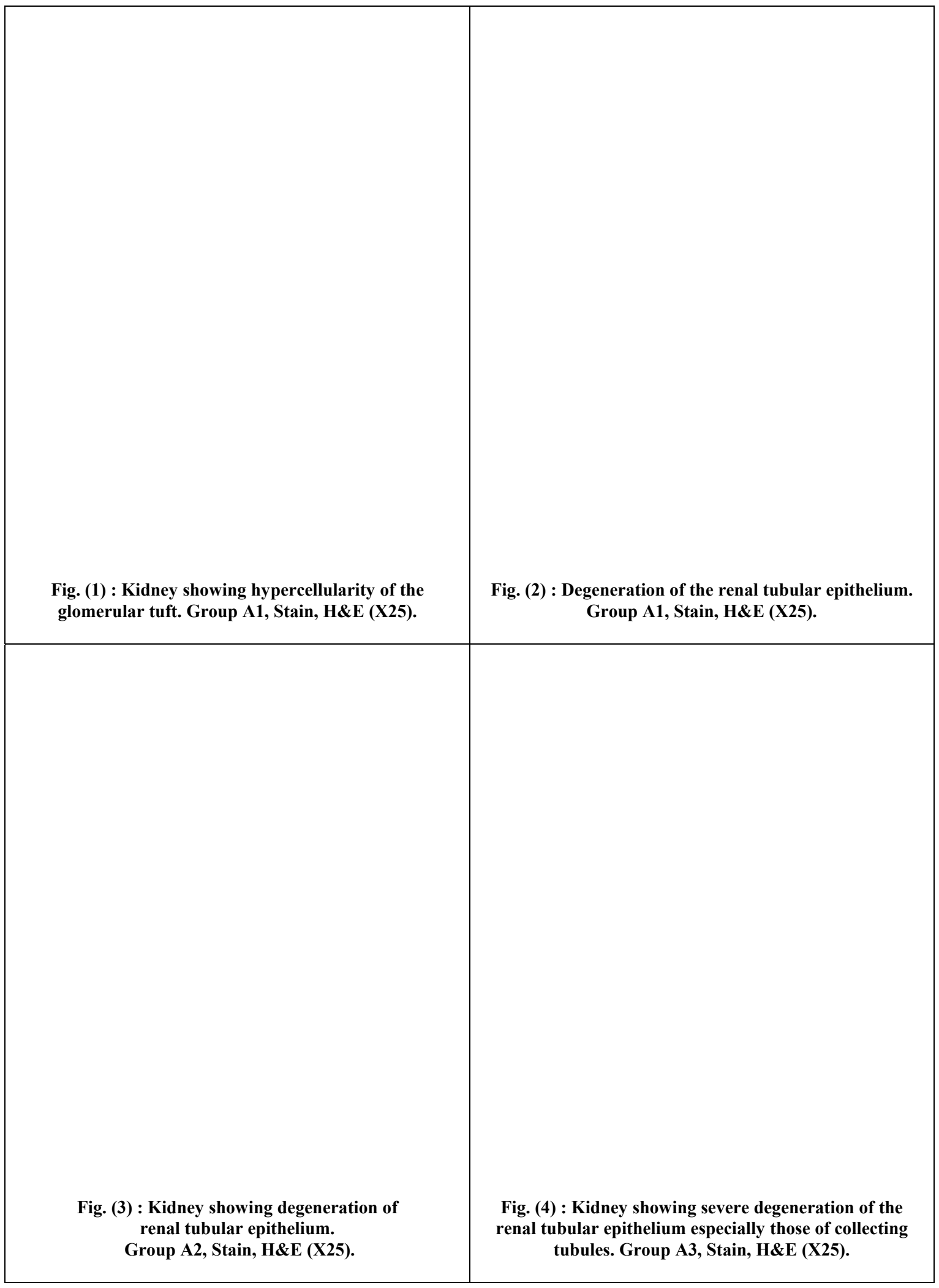




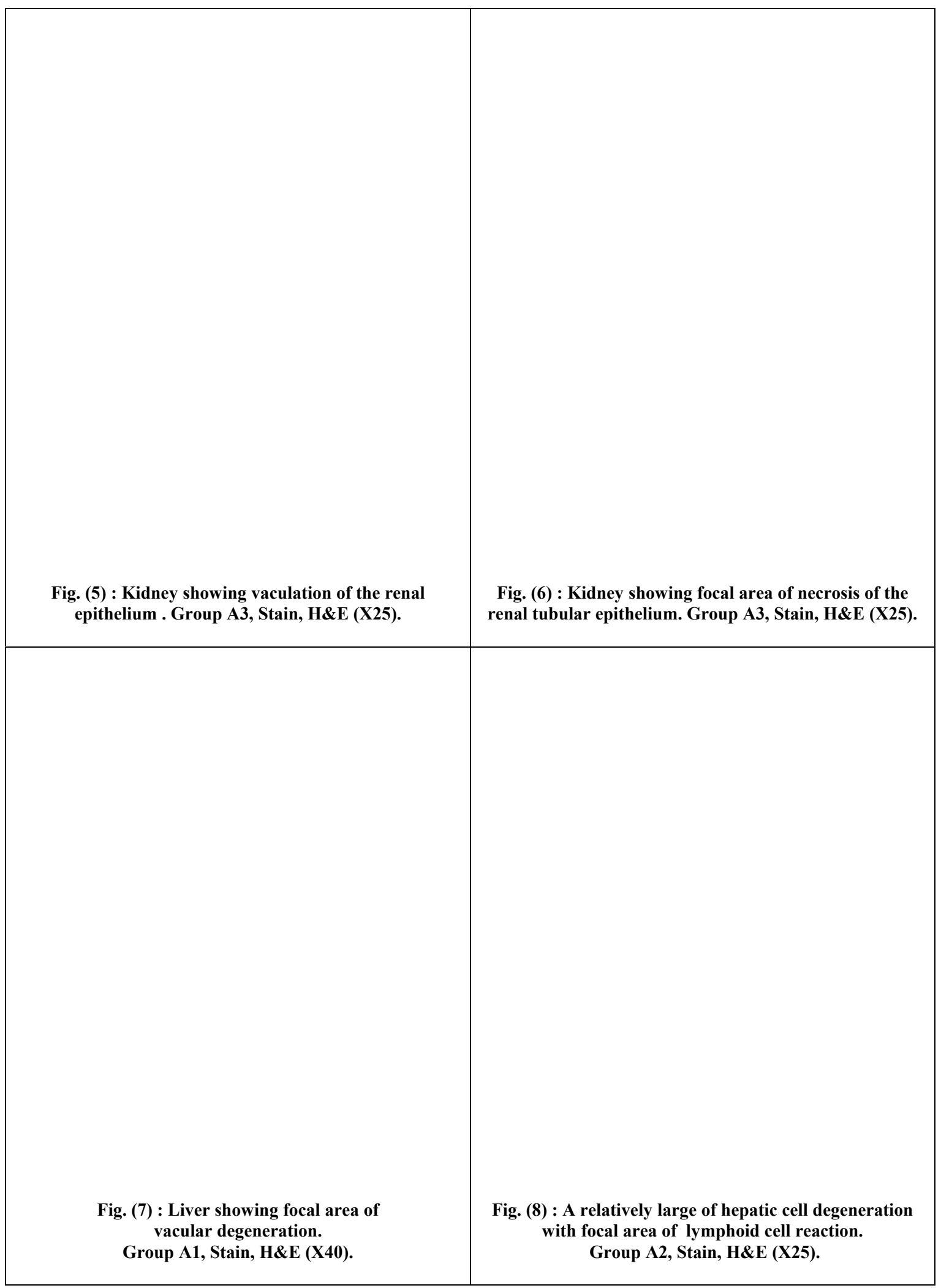




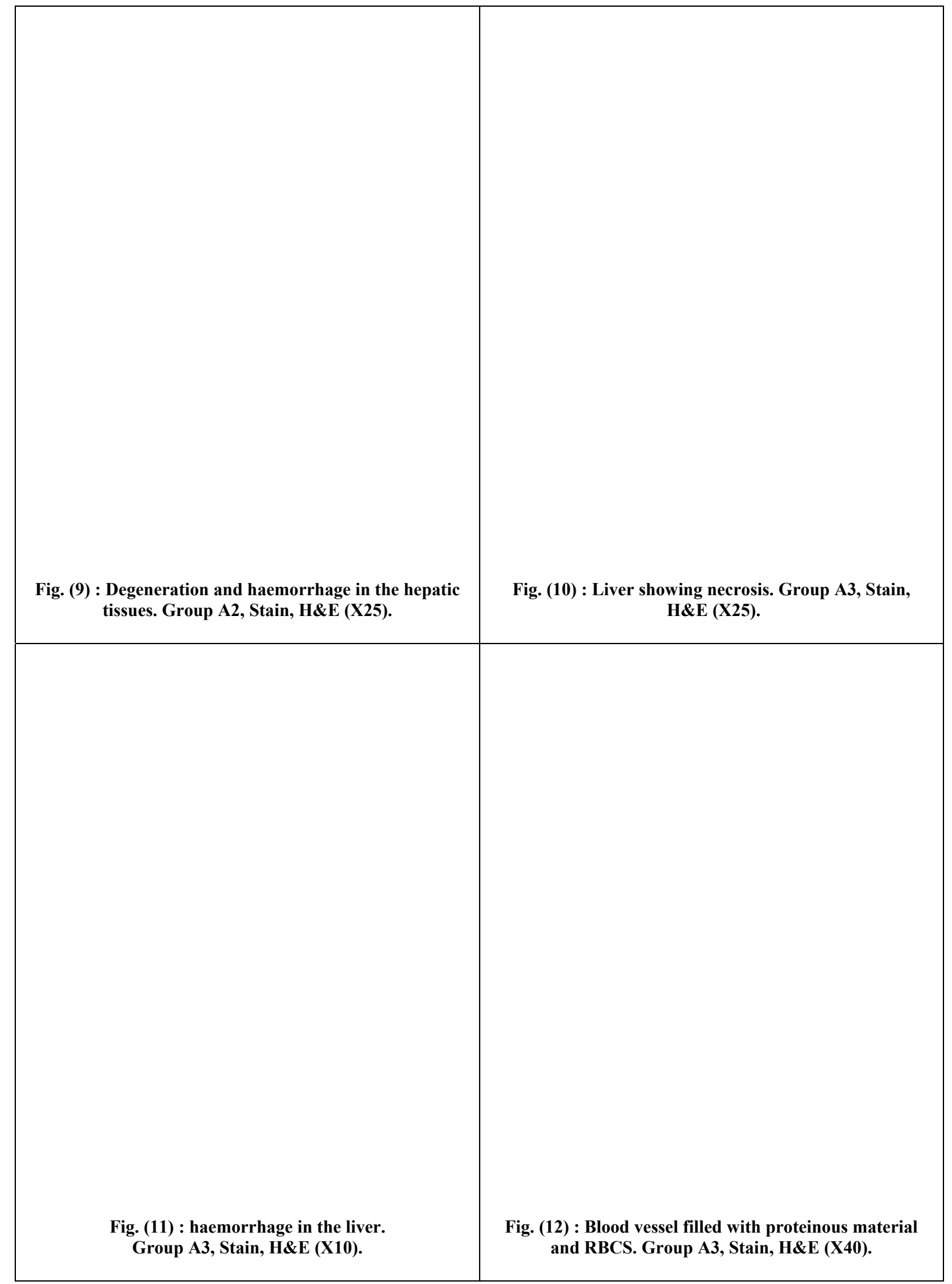




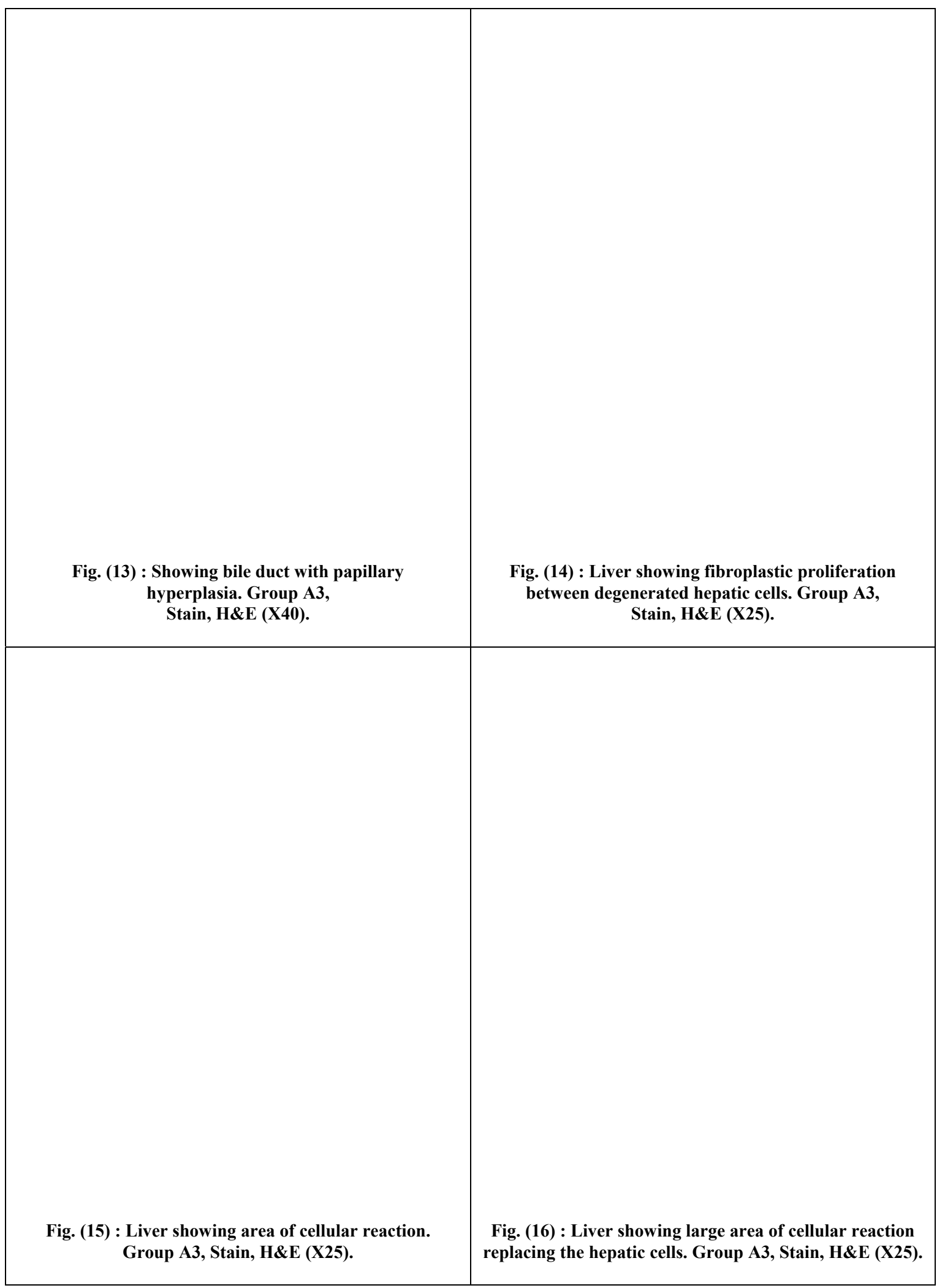




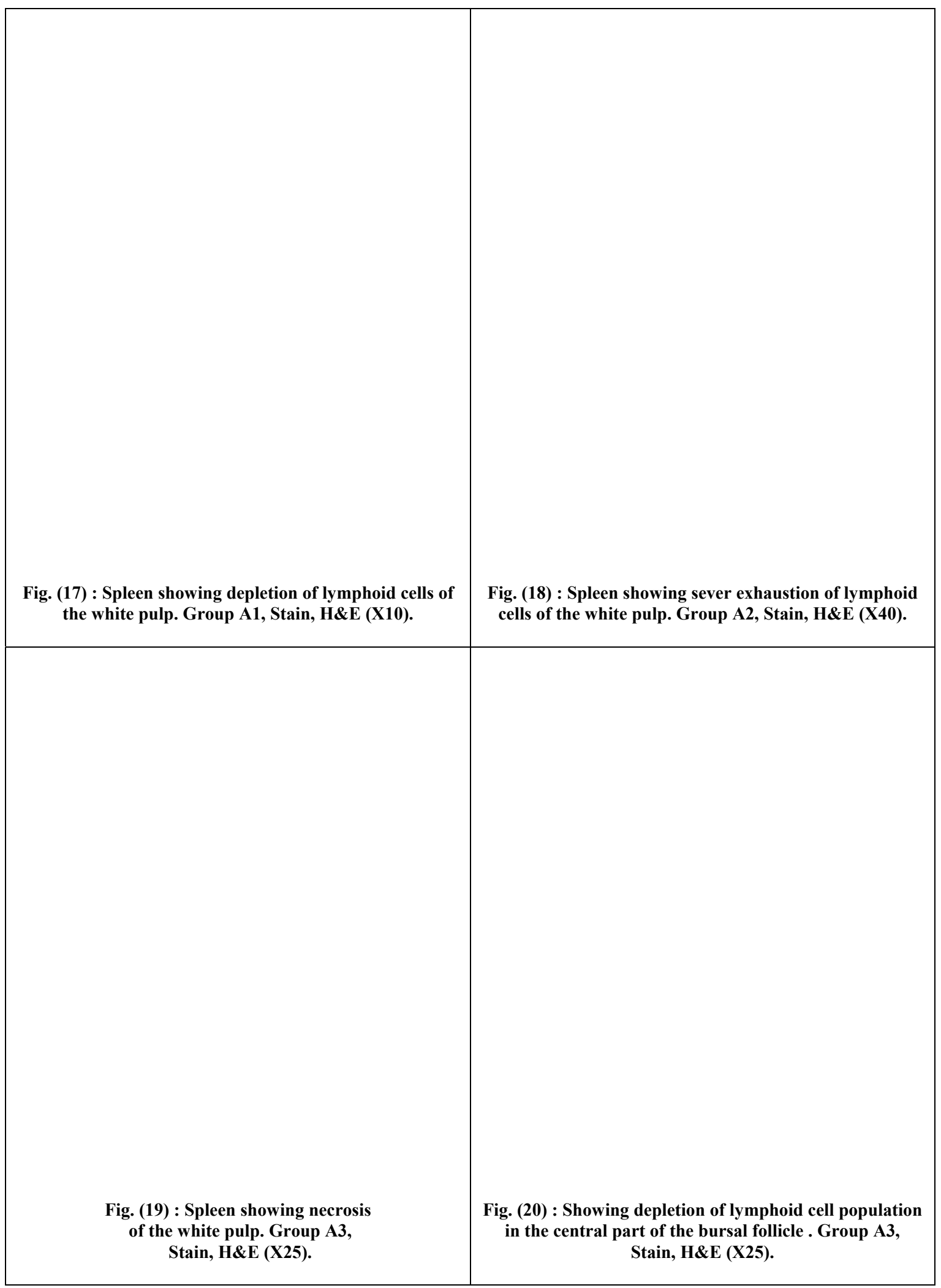




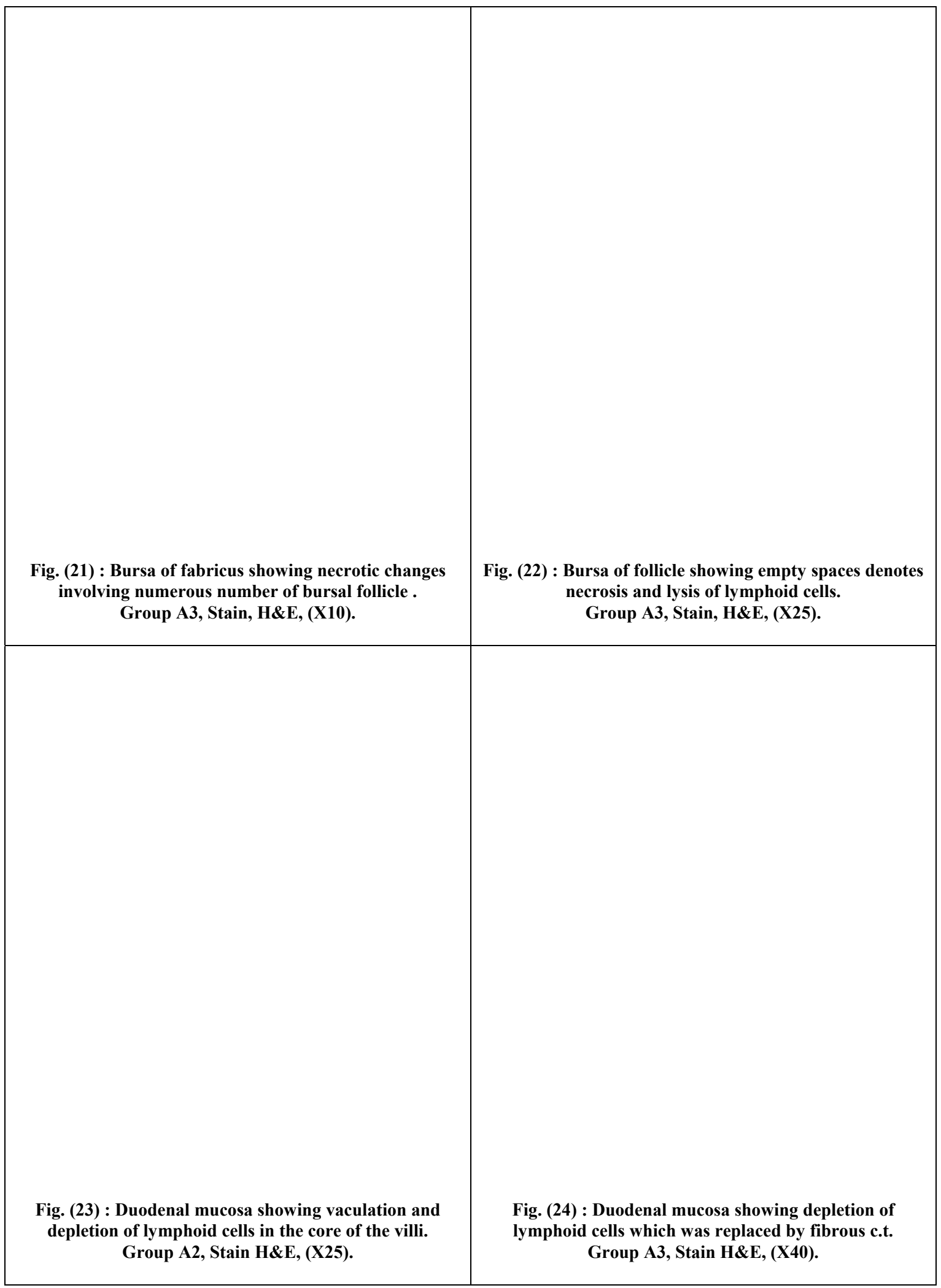




\section{DISCUSSION:}

The results of this investigation indicated that anaemia is one of the most prominent effects caused by exposure to SQO. It could be attributed to deficiency of erythropiotic factor and ascorbic acid, which formed in the renal tubular epithelium. This concept was based upon the fact that renal tubular epithelium showed varying degrees of degenerative and necrotic changes in all tested chickens. Similar results were also observed [12\& 13], who stated that aplastic and haemolytic anaemia were reported in chickens as a manifestation of SQO toxicity and attributed to ascorbic acid deficiency. Ascorbic acid deficiency initiated renal damage resulted in an increase in the capillaries fragility and permeability leading to haemorrhage [14].

The principal site of ascorbic acid synthesis in the chickens was the kidney microsoms [15\&16] supported our opinion. Another explanation for the pathogenesis of anaemia based upon the damage of renal tubular epithelium that was reported in all experimental chickens was given $[17 \& 18]$. They found that SQO when fed to broiler chickens over seven days of the therapy program cause methaemo-globinaemia and alteration in acid-base balance because of inhibition of carbonic anhydrase which happened as a reflect action of the kidneys damage by sulfonamide crystals.

Inhibition of folic acid synthesis due to the renal damage may contributed to the development of anaemia [19\&20].

Haemorrhages were reported in most cases of experimental groups especially those administrated the therapeutic and over dose for long time. These haemorrhages occured most constantly in the skeletal muscles (thigh and breast) and in the parenchymatus organs namely liver, intestine, spleen and kidneys. These results were inagreement with previous reported data [21, 20\&14].

Restoration of normal haemogram in different groups occurred at variable periods. Restoration occured two days after SQO stoppage in group Al. The normal haemogram of A2 and A3 groups was not restored till the end of the experiment.

Anaemia and haemorrhage reported in all experimental groups of chickens might due to the severe damage of the lymphoid tissues from the affected birds' [22].

Restoration of the normal values of total serum protein and albumin occurred at different times in experimental groups. However, those administered therapeutic and toxic dose along the prophylactic one (A2\&A3), the restoration did not occur till the end of the experiment. Restoration of the normal value of total protein in A1 group was considerably delayed as it occurred at 14th day after stoppage SQO. Albumin restoration occurred at 12th day after stoppage of the drug.

Restoration of the normal values of the serum globulin did not occur in all treated groups of chickens (A1, A2\&A3)

Serobiochemical investigation of AST, ALT and $\gamma$ GT revealed an increase in the activity of these enzymes in all chickens whether administered a prophylactic, therapeutic or toxic dose of SQO. Increased level of these enzymes in the serum could 
be resulted from the escape of these enzymes from injured liver cells (ALT\& AST) or from the damaged biliary epithelium $(\gamma \mathrm{GT})$, such result was also obtained [23\&24]. Restoration of such enzymes to the normal values was occurred within different periods in chickens after stoppage of SQO. Restoration was considerably delayed in case of AST in A1, A2 and A3 groups or sometimes did not reoccur till the end of experiment as ALT in the same groups.

Restoration of the normal values of AST and ALT was considerably related to dose and the duration of exposure. It could be also concluded that restoration of these enzymes activities are correlated with the regenerative process observed in liver. Restoration of the $\gamma$ GT normal values is considerably delayed in $\mathrm{A} 1$ and $\mathrm{A} 2$ groups. The normal values of $\gamma \mathrm{GT}$ were not restored until the end of the experiment in A3 group.

Creatine is excreted in the urine of birds before it has been converted to creatinine. The excretion of creatinine may be one reason that its level doesn't provide an accurate measurement of renal function [25]. It has been demonstrated that increased creatinine level occurred only in case of sever kidney damage based on these facts. The level of creatinine was increased only in the serum of group A3 chickens in which severe kidney lesions were reported. Change in the serum creatinine levels in other groups was of no significance value.

Restoration of the normal values of serum creatinine occurred very rapidly after the stoppage of SQO administration even in chickens administered an over dose and related to rapid excretion of creatinine in the urine [25].

Hyperuracemia was demonstrated nearly in the serum of all experimental groups. This hyperuracemia apparently might be due to hepatocellular degeneration and renal damage. This concept was based upon the fact that synthesis of uric acid occurred in the liver and renal tubular epithelium of birds' [26] Such result was also obtained by [27] who stated that hyperuracemia can be expected if the glomerular filtration is decreased to 70 or $80 \%$. The use of nephrotoxic drug might also lead to hyperuracemia [28].

Hyperuracemia observed in group A3 chickens did not restore its normal value till the end of experiment. Other groups' hyperuracemia was restored only after a relatively long period at 16 th day to 18 th day after SQO stoppage. The permanent micromorphological changes in the renal tissues especially in chickens exposed to the toxic dose could explain these findings.

The results of residual analysis revealed that SQO was detectable in muscles, liver and kidneys of all groups at $12 \mathrm{~h}$ post administration. These residual levels continued in elevation till it reached its maximum level at $144 \mathrm{~h}$, which represented the peak of tissue concentration in muscles, liver and kidneys. The residual contents of muscles, liver and kidneys were decreased to the half of its values after SQO stopped in all groups. Residue concentrations were continued to decrease till the nondetectable value $(<1.0 \mathrm{mg} / \mathrm{kg})$. The longest period and the highest concentration of residues were observed in the kidneys followed by liver, and muscles in all groups. A similar result was obtained by [29\&30], as they found the 
maximum level of sulfa residue in tissue at the last day of administration

The residue of SQO was not detected in kidneys, liver and muscles from group A1 at 18th day after stoppage of drug. The kidneys from groups $\mathrm{A} 2$ and $\mathrm{A} 3$ which administered prophylactic, along with therapeutic and overdose of SQO showed the highest concentration of the residual content and the longest withdrawal time where the residues can be detected till the end of our experimental period. This means that kidneys from groups A2 and A3 required more time to be free from the residues. Because of the residual content in the liver of the same groups was less than in kidney by $54 \%$, the period needed for complete withdraw of the residues from the liver was shorter than from the kidney.

The muscles showed lower levels of residual content than in the kidneys and liver of the same groups. It also showed the shortest withdrawal period when compared with those in the liver and kidneys. Residue could not be detected in muscles at the 16th day after stoppage of the drug.

From these results we conclude that the residual content in different organs and the withdrawal time were dose related, it can be correlated well with the duration of SQO administration.

The withdrawal times that observed in our result were 16 days for $\mathrm{A} 1$ group and 18 days for $\mathrm{A2}$ and $\mathrm{A3}$ groups. These groups showed the severest histopathological changes in the liver and kidneys, which can explained the obtained longer withdrawal time. Environmental factors as high temperature might lead to an increase of water consumption that was associated with an increase of drug intake. This will contribute to prolongation of the withdrawal period [31].

Micromorphological changes were varied in its severity and distribution according to the length of administration period and the dose of SQO. The principal lesions were observed in the renal tubular epithelium, these lesions involving degeneration and necrosis of the renal tubular epithelium with pyknosis, karryorhksis and karryolysis of its nucleus. These necrotic changes were observed in A2 and A3 groups' chickens. These changes could be due to the direct action of SQO or might be secondary to hypoxia, which resulted from anaemia. Acute tubular necrosis or degeneration is a primary process; it is an important cause of acute renal failure. The principal causes of acute tubular necrosis and degeneration are eschemia and nephrotoxins [32]. Degenerative changes in the renal tubular epithelium in chickens administered prophylactic dose for a short period or only therapeutic dose were of mild nature and were represented by vaculation and albuminous material.

Histopathological changes involving the renal tubular epithelium, glomeruli, interstitial tissues of similar nature were recorded $[14,33,34 \& 35]$

Amorphous crystalline material was observed in the lumen of the collecting tubules in most cases. In our opinion, this amorphous crystalline material represents precipitates of SQO in the tubular lumen. Renal crystallization of SQO might be occurred as a result of either passive tubular reabsorption of water along osmotic gradients or from renal tubular 
secretion of the drugs.

Sulfamethzine (SFMs) are more soluble in alkaline than acidic solution, thus when the urine $\mathrm{pH}$ decreased, the opportunity of SFMs to precipitate increased. Also a decrease of water consumption increases the chance of crystallization [18]. The presence of crystals in the collecting tubules blocks it and resulted in dilatation and atrophy of the upper part of the nephron, thus lead to permanent changes. These changes might lead to impairment of the renal function which contributed the permanent increase of uric acid in those groups with sever kidneys damage. SFM crystals in the lumen of the collecting tubules, renal papillae, renal pelvic were also reported [14\&34]. Prescience of proteinous material and hyaline casts in the lumen of tubules in groups (A1, A2 and A3) of chickens usually indicates increased glomerular permeability of that nephron. It has been postulated that protein urea associated with proteinous homogeneous material in the lumen of the tubules and also resulted from nephrotic syndrome [14].

Macromorpholgical investigation of the liver from different experimental groups revealed that, detectable gross changes were observed in chickens of all groups (A1, A2 and A3). In which liver was swollen and showed whitish foci response. Lesions of a similar nature were recorded [33].

Hepatocellular degeneration associated with focal area of necrosis were the most common histopathological changes observed in chickens administered prophylactic dose along with therapeutic or toxic dose of SQO. The intensity and distribution varies greatly among different groups of chickens. These changes were extended to involve a massive area of the hepatic parenchyma in those chickens administered toxic dose. Hepatocellular degeneration and necrosis were a manifestation of the toxic effect of SQO on hepatic cells as SFM were eliminated from the body [25]. It might also be due to hypoxia resulted from anaemia observed frequently in all experimental chickens. Binding of SQO to plasma protein of the serum might also play an important role in the disturbance of the metabolism of the hepatic cells leading to degeneration and necrosis [37]. The lesions following SFM therapy attributed to akkergic reaction [38]. In our chickens few cases showed mild eosinophilic cell infiltration in the liver, this indicated that lesions in our experimental birds have no allergic basis.

It appeared that SQO have a deleterious effect on the hepatic cell parenchyma and might lead to hepatic hypofunction. The later was manifested by hypoproteinaemia, hypoalbuminaemia and increased activity to some serum enzymes related to hepatic function. Other groups of chickens administered prophylactic dose for a short period and/or therapeutic dose showed hepatocellular degeneration of mild nature and focal distribution. This degeneration was of vacuolar type. It might due to direct effect of SQO on the hepatic cells and was of primary nature representing a disturbance of metabolism of the hepatic cells. A small focal area of cellular reaction of mononuclear and lymphoid cells type considered secondary manifestation of hepatocellular degeneration. Mild fibro-blastic reactions 
were observed in such cases and represent attempts for healing by reconstitution. Lesions of a similar nature in the liver were listed [13,14,20\&35].

The immune system of chickens is composed of differentiated $B$ - and $T$ lymphocytes. T-lymphocytes are found in the periarteriolar lymphatic sheath of the spleen [39]. However the B-lymphocytes are localized in the germinal centers of the spleen, bursal follicles, as well as along the digestive tract. B-lymphocytes are concerned with antibody production through their differentiation into plasma cells, while T-lymphocytes are involved in the cell mediated immunity [40\&41]. Moreover, histopathological investigation demonstrated that exhaustion of lymphoid population involved essentially B-lymphocytes, as necrotic process was reported in the white pulb of the spleen, bursal follicles, payer's patches of the duodenum and cecal tonsils. SQO administrations for chickens display a suppression of humeral immune responses. This will constitute a serious obstacle against any vaccination programs and enhance the exposure of chickens for secondary viral, bacterial, myotic and parasitic infection.

Immunosuppressive effect reported in most of our experimental groups was manifested clinically by hypoglobunaemia. SFM depress the lymphoid system and immune function in birds resulted in the development of fecal bacterial granuloma in the organs and tissues of the affected birds' [42].

Mitosis and proliferation of the reticuloendothelial cells observed in spleen of chickens seriously affected represent an attempt of compensatory reaction for necrosed lymphoid cells.

The study showed that broiler chickens administered prophylactic and/or therapeutic and toxic dose of SQO for long or short period developed a variable degree of anaemia and hemorrhage in the internal organs and muscles. The most severe pathological lesions were detected in the kidneys followed by the liver. These lesions were correlated with the concentration of residues of SQO in these organs, persistence of its residues and the length of the withdrawal period.

Acute and chronic nephropathy and hepatopathy leading to hypofunction of the kidneys and liver which were manifested clinically by hypoproteinaemia, hypoalbuminemia, hypoglobulinemia, hyperuricemia, increased concentration of creatinine and increased activity of some serum enzymes related to hepatic function (AST, ALT \& $\gamma$ GT). This renal and hepatic hypofunction have an adverse deleterious effect on the productivity of broiler chickens.

The longest withdrawal period was observed in the kidneys followed by liver and muscles. The presence of a relatively higher concentration of residues in the tissues and the relatively long withdrawal period reported in this study are scientific points of considerable importance, as it constitute a potential public health hazard. A strong regulatory action against violators is recommended. A regulatory enforcement in reducing illegal residues is also urgent.

Considering the above mentioned findings, it was recommended that the usage of SQO for combating infectious 
diseases of chickens and as antimicrobial growth promoting factors must be reevaluated, and it is better to resorted to other antimicrobial drug or antibiotics. As it seems that chickens are highly susceptible for SQO adverse effect, excessive dosing must be avoided, an ample supply of water must be provided.

\section{REFERENCES :}

1-Natt, M.P. and Herrick, C.A. (1952) : A new blood diluent for counting the erythrocytes and leucocytes of the chickens. Poult. Sci., 31,735.

2-Cruichshank, R.; Duguid, J. P.; Marmion, B. P. and Swain, R.M.A. (1975): Medical Microbiology. 12 th. Ed. Curchill Living Stone. Edinbugh, London and New York.

3-Schallm, OP.W.; Jain, N.C. and Carrol, E.J. (1979): Veterinary hematology. Lea \& Febiger, Philadelphia.

4-Coles, E.H. (1980): Veterinary clinical pathology.ed., W. B. Saunders Company, Philadelphia and London.

5-Weishelbaum, T.E. (1946): Biochemical determination of total protein Am. J. Clin. Path. 17: 401 .

6-Doumas, B. (1971): Biochemical determination of albumin concentration. Clin. Chem. Acta. 31, 87.

7-Henery, J. (1960): Interpretation of clinical laboratory tests. Am. J. Clin. Path. 34-38.

8-Tietz, N.W. (1994): Text book of clinical chemistry, 2nd ed. W.B. Saunder Co.; Philadelphia, PA, p. 851 .

9-Barham, D.; Trinder, Q. and Analyst.
(1972): Determination biochemically the uriacid metabolites. J. Assoc. Off. Anal. Chem. 97, 142-145.

10-Siest, G.; Henny, J.; Schiele. F. and Uoung, D.S. (1985): Biomedical publications (1985). Inter pretation of clinical laboratory testes. 220-234 .

11-Tishler, F.; Sutter, J.L.; Bathish, J.N. and Hagman, H.E. (1968): Clorometric method for determination of SPM. J. Agric. Food Chem. 16, 50-53 .

12-Sadek, S.E.; Hanson, L.E. and Alberts, J.O. (1955): Suspected drug induced anemias in the chicken. J. Amer. Veterinary Medicine. Assoc. 127: 210203 .

13-Sanger, V.L., Div. M.; Si. Yacowitz H., Moore, D.V.M. (1956): Micropathological changes in experimental hemorrhagic syndrome in chickens fed sulfaquinoxaline and suggested cause of the disease. Am. J. Vet. Res. 17: 766-770.

14-Jubb, K.V.E. and Kennedy, P.C. (1995) : Pathology of domestic animals 3 th. Ed. Academic Press, New York.

15-Duncan, G.G. (1977): Diseases of metabolism. second ed, W.B. Saunders Co. Philadelphia pap. 366-367.

16-Chatterjee, I.B.; Chosh, N.C. and Cuha, B.C. (1961) : Aspects of ascobic acid biosynthesis in animals. Ann. New York Acad. Sci. 92: 36-56.

17-William, W.J. (1972): Hematology. New York, Mc Graw-Hill, P.209 .

18-Bevill, R.F. (1975): Sulfonamides metabolism, excretion in animal tissues. $1^{\text {St }}$. Quartery Report FDA 71-69.

19-Scott, M.C.; Nesheim, M.C. and Young, R.J. (1982): Nutrition of the chicken. 
ML Scott, P.O. Box 816, Ithaca, NY 1985, USA .

20-Jordan F.T. (1990): Sulfonamides induce anemia through inhibition of folic acid. Poultry diseases. Third ed. Great Britan 326-327.

21-Nordstoga, K., Helgebostad, A.; Lottsgard, G. and Storwoken, H. (1970): Vesicle hemorrhage in male Aleutian mink caused by hypersensitivity to sulphadimidine. Acta. Vet. Scand. 11: 481 .

22-Faddul, G.P.; Amato, S.V.; Sevian, M. and Fellow, G.W. (1967) : Studies on intolerance to sulfaquinoxaline in chickens. Avian Dis. 11, 226.

23-Ford, E.J.H. and Lawerence, J.A. (1965): Hepatic and serum changes following reported administration of small amount of cabon tetrachloride in sheep. J. Comp. Path. 75, 185.

24-Zimmerman, H.J. (1978): Hepatotoxicity. Applet on century. Gro Fts. New York. 180-187.

25-Bell, D.J. and Freman, B.M. (1971): Physiology and Biochemistry of the Domestic Fowel. New York, Academic Press.

26-Ghoust, (1972) : Relative importance of liver and kidney synthesis of uric acid in chickens. Can. J. Physiol. Pharm. 50: 936-939.

27-Gylsroff, I. And Crimm, F. (1987) : Hepatopathy associated with biochemical parameters induced by sulfaquinoxaline. Vogelkrankheiten. Stuttgart, Eugenul-mer. pp. 133-146.

28-Anderson, C.B. (1989): Determination of creatinine in kidney, plasma and serum protein concentration by refractory and biuret method. Avian. Dis. 33:93-96.

29-Rana, R., Akhtar, M.S. and Nawaze, M. (1993): Residues of sulfaquinoxaline in poultry products. Pakistan. Vet. J. 13 (4): 161-166 .

30-Nawas, R. Ahmed, R. Pervaiz, A. and Muhammad, N. (1996): Residues of sulfadimethoxine in blood, eggs and tissues of poultry birds. Pakistan Veterinary J., 16 (4) : 180-185.

31-Gurda, F.R.; Carlond, T. and Paolo. (1973) : Pathology of acute and chronic poisoning in the presence of residues and the therapeutic activity of the combina-tion of dieveridine and sulfadimidine. Ser. Medicine Veterinary, 2: 130-172.

32-Well, G.A.H.; Hebert, C.N. and Robins, B.C. (1980): Renal cysts in pigs prevalence and pathology in slaughtered pigs from a single herd. Vet. Rec. 106: 532-635.

33-Morgan, R.V. Bachrach, A.Jr. (1982) : Histopathological examination to lesions in hepatorenal tissues due to sulfaquinoxaline administration. J. Am. Veterinary Ass. 180, 432-434.

34-Jones, T.C. and T,C. and Hunt, R.D. (1983) : Veterinary pathology $5^{\text {th }} \cdot$ Ed. Lea \& Febiger, Philadelphia.

35-Clank, B.W.; John, H.; Barnes; Beard, C.W.; Red, W.M. and Yoder, H.W.Jr. (1994): Diseases of poulty in United States of America. $9^{\text {th }}$. Ed. 863-864.

36-Harper, M.A. (1973): A review of pathological chemistry 14 th. Ed. Large Medical Publication, Los Altos, California. 
37-Davis, S.F.M. (1954): Sulfonamides Pois-oning in Chickens treated for coccidiosis. Proc. Tenth World's Poultry Congress.

38-Lodge, K.V. and Woodcock, A.S. (1954) : Acute necrosis of liver following sulfonamide therapy in patients with leukemia. Am. J. Path., 361-373.

39-Wara, D.A.; Goldstein; Doyle, N. and Ammanna, A. (1975): Thrymosin activity inpatient with cellular immune deffeciency.

40-Eisen, M.N. (1973): In Microbiology (Davis B.D. Dulbeco. M.N. Eisen. Cinsher. M.S.;W.B. Wood and Macarty) Harper and Row, Hagerstown,
Maryland pp. 351-510.

41-Giambrone, J.J.; Danaho, J.P.; Dawe, D.L. and Eidson, C. S. (1977): Specific suppression of the bursa dependent immune system of chicks with infectious bursa disease virus. A.J. of Veterinary Res. 38: 581-583.

42-Blom, A.L. (1975): Plasma half-lives and the excretion into egg-white and eggyolk of three sulfonamides and pyrimethamine after medication of laying hens. Acta Pharmacol. Toxicol. 37: 79-83. 
تقييم التعرض الممتد للسلفاكينوكز الين على أداء وظائف الكبد والكلى في كتاكيت إنتاج اللحم بالإشارة إلي بقايا الاواء باء بالأنسجة

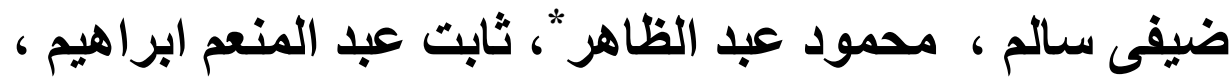

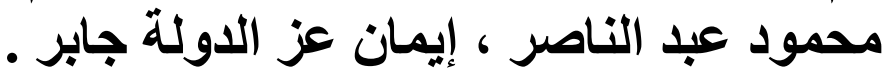

قسم الطب الثرعى والسموم- قسم الباثولوجيا والباثولوجية الإكلينيكية.

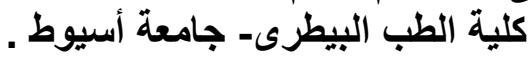

تم دراسة تأثير السلفاكينوكزالين على مكونات الام ووظائف الكبد والكلى فى كتاكيت انتاج اللحم عند تعرضها

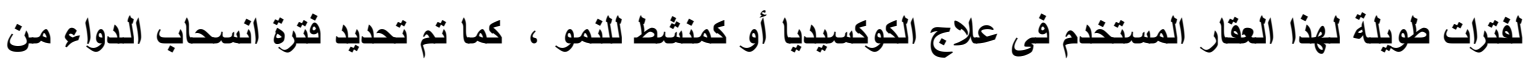

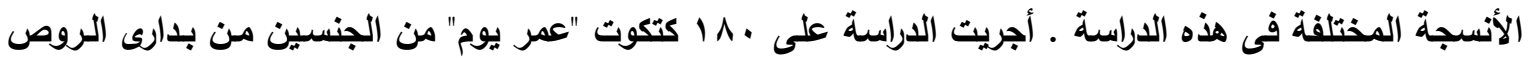

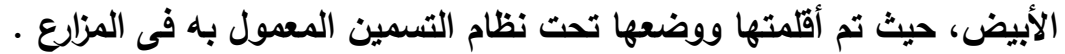
أعطيت الكتاكيت جرعة وقائية من السلفاكينوكزالين (اجم/تلتر ماء) اعتباراً من اليوم الأول وحتى اليوم الواحد

$$
\text { والثثلاثين ، وقسمت بعدها إلى ثلاث مجموعات : :- }
$$

المجموعة الأولى (A1) : استمرت فى تثناول الجرعة الوقائية حتى اليوم الثالث والثثلاثين ثم توقف تناول العقار لمدة يومين ثم أعطيت الطيور العقار لددة ثلاثة أيام أخرى.

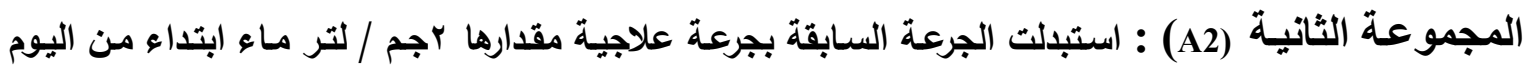
الثاني والثلاثين ثم توقف تناول العقار لمدة يومين ثم أعطيت الطيور العقار لمدة ثلاثة أيام أخرى.

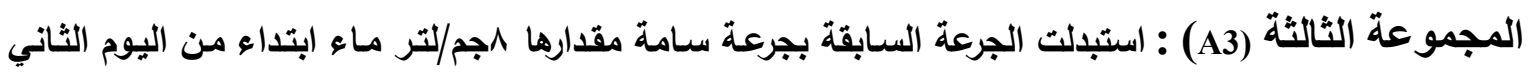
والثلاثين ثم توقف تناول العقار لمدة يومين ثم أعطيت الطيور العقار لمدة ثلاثة أيام أخرى.

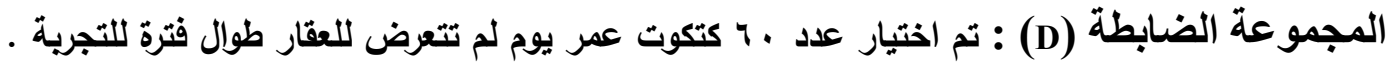
وقد أوضدت الدارة حدوث أنيميا فى مجموعات الاجاج المختلفة بالإضافة إلى زيادة نثاط إنزيمات الكبد ،

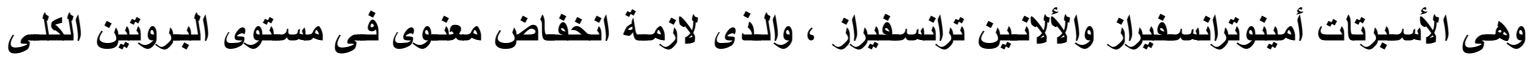
لمصل الام والمصاحب بزيادة ملحوظة في الكرياتينين والحمض البولى الدالة على انخفاض وظائف الكلى.

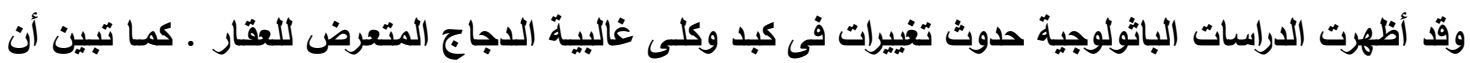

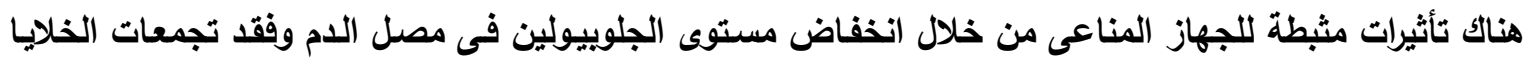

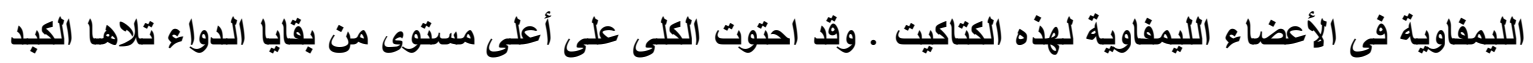

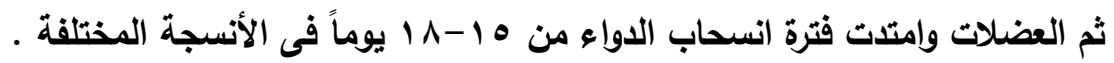
تبين مما سبق أن تعرض الدجاج للسلفاكينوكزالين لفترات طويلة قد أدى إلى تغير فى وظائف الكبد والكلى مما

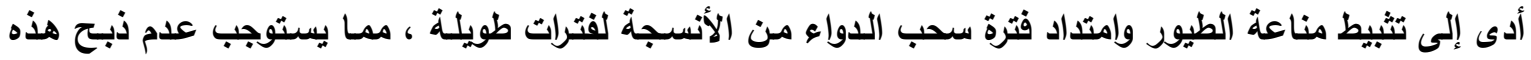

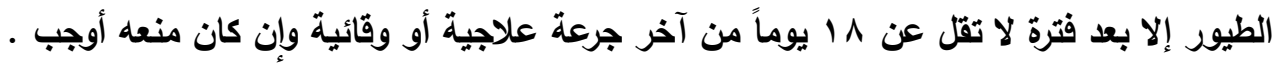

